\title{
A residential energy management system with bi-level optimization-based bidding strategy for day-ahead bi-directional electricity trading
}

\author{
M.S.H. Nizami ${ }^{\mathrm{a}, *}$, M.J. Hossain, B M Ruhul Amin, Edstan Fernandez \\ ${ }^{a}$ School of Engineering, Macquarie University, NSW 2109, Australia.
}

\begin{abstract}
Bi-directional electricity trading of demand response (DR) and transactive energy (TE) frameworks allows the traditionally passive end-users of electricity to play an active role in the local power balance of the grid. Appropriate building energy management systems (BEMSs), coupled with an optimized bidding strategy, can provide significant cost savings for prosumers (consumers with on-site power generation and/or storage facility) when they participate in such bi-directional trading. This paper presents a BEMS with an optimization-based scheduling and bidding strategy for small-scale residential prosumers to determine optimal day-ahead energy-quantity bids considering the expected cost of real-time imbalance trading under uncertainty. The proposed scheduling and bidding strategy is formulated as a stochastic bi-level minimization problem that determines the day-ahead energy-quantity bids by minimizing the energy cost in the upper level considering expected cost of uncertainty, whereas a number of lower-level sub-problems ensure optimal operation of building loads and distributed energy resources (DERs) for comfort reservation, minimization of consumers' inconveniences and degradation of residential storage units. A modified decomposition method is used to reformulate the nonlinear bi-level problem as a mixed-integer linear programming (MILP) problem and solved using 'of the shelf' commercial software. The effectiveness of the proposed BEMS model is verified via case studies for a residential prosumer in Sydney, Australia with real measurement data for building energy demand. The efficacy of the proposed method for overall financial savings is also validated by comparing its performance with state-of-the-art day-ahead scheduling strategies. Case studies indicate that the proposed method can provide up to $51 \%$ and $22 \%$ cost savings compared to inflexible non-optimal scheduling strategies and deterministic optimization-based methods respectively. Results also indicate that the proposed method offers better economic performance than standard cost minimization models and multi-objective methods for simultaneous minimization of energy cost and user inconveniences.
\end{abstract}

Keywords: Demand Response,, Building Energy Management System, Distributed Energy Resources, Mixed-Integer Programming, Bi-level Optimization

\section{Introduction}

Residential prosumers, i.e. consumers with on-site distributed generation (DG) and distributed energy resources (DER), are increasing in numbers due to advances in affordable electrical storage and generation technologies, favorable regulations, and attractive incentive programs. Besides, the evolving electricity market structures allow prosumers to participate in different market-based schemes for cost reduction including demand response (DR) and transactive energy (TE) framework. In addition to that, modern residential buildings are now coupled with various controllable electrical loads, such as electric vehicles (EV) with the vehicle to grid (V2G) and vehicle to building (V2B) functionalities, heat pumps, electric heating, ventilation,

\footnotetext{
* Corresponding author

URL: sohrab.nizami@hdr.mq.edu.au (M.S.H. Nizami), jahangir.hossain@mq.edu.au (M.J. Hossain), ruhul.amin@students.mq.edu.au (B M Ruhul Amin), edstan.fernandez@hdr.mq.edu.au (Edstan Fernandez)
} 


\begin{tabular}{lll}
\multicolumn{2}{l}{ Nomenclature } \\
\multicolumn{2}{l}{ Indices and Sets } & $\rho^{c \leftrightarrow d}$ \\
$i \in \mathbf{T}$ & Time periods & $C^{B}$ \\
$j \in \mathbf{M}$ & Flexible devices & $\rho^{d c m}$ \\
$k \in \mathbf{N}$ & Power levels of flexible devices & $\theta^{\text {set }}$ \\
$h \in \mathbf{H}$ & Set of thermal loads & $P^{f l}$ \\
$p \in \mathbf{P}$ & Building surfaces (walls and roof) & $P^{g}$ \\
$q \in \mathbf{Q}$ & Windows and doors of the building & $\overline{P^{t h \prime}}$ \\
$\mathbf{L}, \mathbf{D} \subset \mathbf{M}$ & Set of time-shfitable flexible loads & \\
& and flexible DER units
\end{tabular}

$\mathbf{L}_{\mathbf{n i}} \subset \mathbf{L} \quad$ Set of non-interruptible loads

$\mathbf{T}_{\mathrm{ts}} \subset \mathbf{T} \quad$ Desired operating window for time-shiftable flexible loads

$s \in \mathbf{S} \quad$ Set of scenarios

\section{Parameters}

$\Delta_{T} \quad$ Duration of 1 time period [hour]

$E_{j}^{r e q} \quad$ Energy consumption requirement of load $j[\mathrm{kWh}]$

$\rho^{\text {int }} \quad$ Penanlty cost coefficient for interruption[\$/interruption]

$\kappa_{j} \quad$ Coefficient of performance (CoP) of thermal load $j$

$Q^{i n t} \quad$ Internal heat gain of the building $[\mathrm{kW}]$

$\beta, R \quad$ Heat transfer coefficient $\left[\mathrm{W} / \mathrm{m}^{2} \mathrm{~K}\right]$ and radiation heat resistance $\left[\mathrm{m}^{2} \mathrm{~K} / \mathrm{W}\right]$ of building surfaces

A Surface area $\left[\mathrm{m}^{2}\right]$

$\theta^{\text {out }} \quad$ Outdoor temperature $\left[{ }^{\circ} \mathrm{C}\right]$

$\alpha, \tau \quad$ Radiation absorption and transmission coefficient of the building surfaces

I Solar radiation on the building surfaces/windows/doors $\left[\mathrm{KW} / \mathrm{m}^{2}\right]$

$\rho, V, C_{b} \quad$ Density $\left[\mathrm{kg} / \mathrm{m}^{3}\right]$, volume $\left[\mathrm{m}^{3}\right]$ and specific heat capacity $\left[\mathrm{J} / \mathrm{kg}^{\circ} \mathrm{C}\right]$ of the air in the building

$P^{c}, P^{d} \quad$ Charging and discharging power for storage $[\mathrm{kW}]$

$\eta^{c}, \eta^{d} \quad$ Charging and discharging efficiencies for storage $[\mathrm{kW}]$

$E^{m} \quad$ Maximum storage capacity [kWh]

$\overline{S o C_{j}}, \underline{S o C_{j}}$ Maximum and minumum state of charge (SoC)

$\overline{D o D_{j}}, D o D_{j}$ Maximum and minumum depth of discharge (DoD)
Penanlty cost coefficient for frequent charge-discharge cycles

Battery replacement cost

Thermal discomfort coefficient

Thermostat set-point temperature

Fixed-load demand [kW]

Solar PV generation $[\mathrm{kW}]$

Maximum power demand of thermal load $[\mathrm{kW}]$

\section{Variables}

$x, y$

Binary decision variable $\in\{0,1\}$

$C_{\text {int }}$

Penalty cost for interruption

$Q^{\text {gen }}$

Heating/cooling power generation by thermal loads $[\mathrm{kW}]$

$Q^{\text {con }}$

$Q^{r a d}$

$Q^{c s}$

$Q^{c w}$

$\theta$

$Q^{r s}$

$Q^{r}$

$Q^{r w}$

Heat exchanges in conduction $[\mathrm{kW}]$

Heat exchanges in radiation $[\mathrm{kW}]$

Heat exchanges due to thermal conduction via external surfaces of the building $[\mathrm{kW}]$

Heat exchanges due to thermal conduction via windows and doors $[\mathrm{kW}]$

Indoor temperature $\left[{ }^{\circ} \mathrm{C}\right]$

Heat contribution of solar radiation on the opaque surfaces of the building $[\mathrm{kW}]$

Heat contribution due to solar radiation transmitted through windows and doors $[\mathrm{kW}]$

$\sigma_{i, j}, \delta_{i, j} \quad$ State of charge (SoC) and depth of discharge (DoD) of storage units

$\Phi_{i, j} \quad$ Marginal cycle aging degradation of storage units

$C_{D o D}, C_{c \leftrightarrow d}$ Battery degradation cost for nonoptimal DoD and frequent chargedischarge cycles

$P^{t h \prime} / P^{t h} \quad$ Power demand of thermal load with/without VESS

$P^{\text {thc }}, P^{\text {thd }} \quad$ Charging and discharging power of VESS

$C_{d c m} \quad$ Thermal discomfort cost

$2 P^{+}, P^{-} \quad$ Day-ahead bids (demand and supply) $[\mathrm{kW}]$

$\Delta P^{r t} \quad$ Real-time imbalance bids $[\mathrm{kW}]$ 
and air-conditioning (HVAC) systems, etc. As a result, building energy management systems (BEMS), with appropriate load scheduling and resource optimization strategy, offer significant cost savings to the prosumers by utilizing the flexibilities of these controllable residential loads and DER units for bi-directional electricity trading of DR and TE frameworks [1, 2].

Traditional DR programs influence responsive consumers and prosumers to alter their consumption profile according to the need of utility companies using price or incentive signals. It allows them to utilize the demand-side flexibilities for peak load reduction, which in return reduces the power generation and operation cost [3]. On the other hand, TE is an advanced variant of DR, where consumers and prosumers can actively negotiate the energy to be consumed or supplied for each market interval [4]. In addition to peak-load reduction, TE offers several grid-assistance services including grid-congestion management [5, 6] and local voltage regulation [7. Moreover, the local peer-to-peer (P2P) electricity trading of the TE framework reduces the stress on grid supply 8 and offer significant profits to prosumers [9. However, TE is still in the infancy phase without any established standards, and most TE markets do not allow small-scale prosumers to actively negotiate the tariff for energy transactions, rather they are aggregated via a local transactive aggregator thereby limiting their cost-reduction potential [4, 10, 11.

Significant research efforts have been made towards developing efficient BEMS for bi-directional electricity trading of DR and TE frameworks. For example, the bi-directional DR trading-based energy management methodologies in 12, 13, 14, 15, optimally schedule and manage controllable building loads and energy resources to reduce electricity bills for the consumer, whereas the methodologies in 2, 16, 17, also incorporated user inconveniences and discomfort while minimizing energy cost for the consumers. Several studies proposed BEMS methodologies for energy trading or sharing in the TE framework. For example, the energy management methodologies in 11, 18, 19 reported significant cost savings for participating consumers in a local TE trading, where consumers trade demand flexibility and excess energy from building resources during grid-congestion periods. The BEMS presented in [20] trades the flexibilities of the HVAC system in a local transactive market for peak-load reduction, demand shifting, and conservation of HVAC demand of a commercial building. The flexibilities of thermostat-controlled building loads are also used in [21] to trade demand-flexibility in the real-time transactive market and reduce electricity costs for the consumer. The authors in [8, 22, 23 proposed methods to manage residential storage units for P2P energy trading or sharing in a local community with a focus on electricity bill reduction for the prosumers. Local P2P sharing is also adopted in the energy management methodologies in [9, 24. The energy exchanges between neighbors are optimized in 9 based on their demand, battery state of charge and excess generation from on-site solar photo-voltaic (PV) systems. On the other hand, the BEMS in [24] schedules the building resources in the neighborhood resulting in reduced electricity bills for the consumers, efficient utilization of neighborhood PV generation and reduced peak to average ratio (PAR) on the aggregated demand profile. Local TE trading is explored for microgrids in several research studies [22, 25, 26]. The responsive building loads and generators of the microgrid participate in a local market in [25] to procure or sell energy for the cost minimization of the consumers. On the other hand, P2P trading is used in [26] for the local power balance of the microgrid, which reduces energy export from the utility grid and facilitates higher penetration of renewable energy resources.

However, cost minimization approaches of $[8,9,12,13,14,15,18,21,22,24,25$ can often result in user inconveniences when demand flexibility is leveraged to maximize profits out of bi-directional energy exchange of DR and TE schemes. For example, flexible operation of thermostat-controlled appliances can cause thermal discomfort for the user as such approaches stretch the indoor temperature to maximum or minimum thresholds most of the time to reduce heating and cooling demand (as seen in 13, 14). Such thermal discomfort is not considered in [11, 12, 13, 14, 17, 20, 21] when a flexible operation of thermostatcontrolled building appliances is utilized for reducing electricity cost. On the other hand, the methodologies in [15, 16, 25] involve curtailment or deferral of flexible loads, which can cause user dissatisfaction, hence need to be incorporated into the BEMS models for actual welfare maximization of the consumer. On top of that, the models in $8,9,12,13,14,15,16,17,18,22,24$ utilizes the flexibility of battery storage units to minimize electricity bills when participating in bi-directional trading-based DR or TE framework. However, such approaches often lead to irregular and frequent charge-discharge cycles for the storage units thereby causing their lifetime degradation [27, which needs to be incorporated into the BEMS model. The 
user dissatisfaction for load curtailment or interruptions are considered in [17] by imposing a constraint to limit it within the desired level, however, such measure may not lead to most optimum operation. On the other hand, the interruptions of flexible loads are penalized in [16, 19, which are minimized along with the energy cost for the consumer. Similar approaches are taken for thermal discomfort reduction in 19, 26 and minimization of storage degradation in 19, 23. However, such a single-objective-based approach by adding completely different cost terms may not provide the most optimal electricity cost scenario as it reduces combined cost (including penalty cost for interruptions and thermal discomfort) instead of minimizing actual electricity cost. On the other hand, the multi-objective optimization-based model of 2] for simultaneous minimization of electricity cost, thermal discomfort, user inconveniences, and storage degradation is highly dependent on the weighting coefficient used for different objectives [28. In addition to that, most bi-directional energy markets include a day-ahead market and a real-time imbalance market, where the participants commit to energy consumption and supply schedule for the next day in day-ahead stages, and trades any imbalances in the real-time [29, 30. In this regard, deterministic approaches can often expose prosumers to higher tariff fluctuations in real-time imbalance market. Several studies proposed stochastic and robust optimization models to incorporate uncertainty in the day-ahead setting [29, 30, 31, however, these methodologies aggregate the demand-side flexibilities of small-scale prosumers and active consumers via an aggregator, who trades energy on behalf of the prosumers and control their building loads and resources. Such methodologies can often raise privacy concerns and prosumers are not provided with full-decision making authority, thus discouraging their active engagement and often fail to utilize their full flexibility [19, 20, 32,

This paper focuses on addressing the research gaps found in the literature by developing a comprehensive BEMS model for bi-directional energy trading that determines optimal day-ahead bids by minimizing the electricity cost while considering user inconveniences in terms of dissatisfaction for load interruptions, thermal discomfort for thermostat-controlled loads, and degradation of storage units. It is considered that the prosumer participates in a day-ahead energy market, and trades real-time imbalances from day-ahead market commitment in a local transactive market that operates in real-time [19. A BEMS model is presented in this paper for small-scale residential prosumer with day-ahead scheduling and bidding strategy that determines the day-ahead energy-quantity bids while considering the expected imbalance cost for the uncertainties. Contrary to most BEMS for bi-directional trading, the bidding model is formulated as a two-stage stochastic bi-level optimization problem where the electricity cost is minimized in the upper level to determine the day-ahead energy bids of the building for each market interval considering uncertainty in demand, generation, and market prices. The inconveniences and thermal discomfort of the consumer have been modeled as optimization subproblems in the lower level along with storage degradations. The use of bi-level optimization allows incorporating discomfort, inconvenience and degradation cost as lower-level subproblems compared to treating them as equivalent objectives as in single-level formulations. As a result, the proposed methodology can minimize the actual energy cost subject to minimum inconveniences, discomfort and storage degradation. Therefore, it diminishes the need for adding different cost terms into a single objective as in [16, 19, 23] or use of multi-objective optimization as in [2]. The key contribution of this paper can be summarized as:

- An improved BEMS model is developed for small-scale residential prosumers to trade energy in a day-ahead energy market considering the expected cost of imbalance-trading in a real-time market.

- A novel transactive bidding model is proposed based on two-stage stochastic bi-level optimization that minimizes the energy cost subject to minimum consumer inconvenience and discomfort while determining energy bids.

- A comprehensive thermal model is developed for the building with a virtual energy storage system (VESS) to represent consumer's flexibility and thermal discomfort for the operation of thermal loads.

- The cycle-aging degradation of the storage units are incorporated into the BEMS considering different stress factor on battery life to minimize the actual cost for the consumer including battery degradation costs. 
The remainder of the paper is organized as follows - the mathematical model of the proposed BEMS is discussed in Section 2, the proposed day-ahead scheduling, and bidding optimization model is presented in Section 3, case studies, and numerical results are discussed in Section 4 to evaluate the effectiveness of the proposed BEMS methodology, which is followed by the conclusion of the paper as given in Section 5 .

\section{The BEMS model}

A comprehensive building energy management system (BEMS) is proposed in this paper that coordinates and manages the operation of all the electrical loads and DERs of the building.

The power demand of non-flexible electrical appliances is considered non-controllable and such loads are termed as fixed loads that include basic lighting loads, television, fridge, cooking appliances, steam iron, etc. The BEMS model ensures uninterrupted operation of the fixed loads whenever the user switches them on, therefore, the power demand for the fixed load is considered constant for the BEMS. The power generation from on-site distributed generation (DG) units (e.g. rooftop solar photovoltaic system) is also uncontrollable as it depends on external factors such as weather condition, temperatures, etc. The BEMS aims at maximum utilization of DG power, which is also considered fixed for energy management system similar to fixed load demand.

However, the power consumption of some building loads can be shifted in time without affecting the user comfort such as washing machine, dishwasher, pool pumps, etc. These building loads are categorized as flexible loads in this paper. In addition to that, the charging and discharging of electrical storage units of the building can be controlled without violating their operational constraints. The term "flexible device" is used in the remainder of this paper to indicate flexible loads and controllable DERs (e.g. electrical storage units) of the building. On the other hand, the thermostat-controlled building loads are considered as thermal loads and a thermal model of the building is proposed with a virtual energy storage system (VESS) for flexible operation of thermal loads.

\subsection{Binary decision variable and operating states of flexible devices}

Two operating states are considered for flexible devices- ON and OFF (or idle) and a binary decision variable, $x$ is used to indicate the operating states of the devices. Here, $x_{i, j}^{k}=1$ if the device $j \in \mathbf{M}$ is operating (ON) with power level $k \in \mathbf{N}$ during period $i \in \mathbf{T}$ and $x_{i, j}^{k}=0$ means that the device $j$ is switched OFF or at idle state at time $i$ for any value of $k$. A device can only operate in one power level at a time. This is represented in the BEMS model by setting decision variable constraint as follows:

$$
\sum_{k \in N} x_{i, j}^{k} \leq 1 \quad \forall i \in \mathbf{T}, j \in \mathbf{M}
$$

Another binary decision variable, $y$ is used to indicate the change in operation states for flexible devices. Here, $y_{i, j}^{k}=1$ only when flexible load $j \in \mathbf{L}$ changes its operating states from OFF to ON for $k=1$ as it indicates that load $j$ switches ON from OFF state in period $i$ and starts its first power cycle with first power level. On the other hand, $y_{i, j}^{k}=1$ if flexible DER unit $j \in \mathbf{D}$ changes operating status from idle to charging (for $k=1$ ) or discharging (for $k=2$ ). Therefore, the condition for $y$ can be written as follows:

$$
\begin{aligned}
y_{i, j}^{k} & =1 ; \text { if } x_{i-1, j}^{k}=0 \& x_{i, j}^{k}=1 & \forall i, \forall k=1, \forall j \in \mathbf{L} \\
& =1 ; \text { if } x_{i-1, j}^{k}=0 \& x_{i, j}^{k}=1 & \forall i, \forall k \in\{1,2\}, \forall j \in \mathbf{D} \\
& =0 ; \text { Otherwise } &
\end{aligned}
$$

\subsection{Time-shiftability of flexible loads}

The operation of flexible loads can be shifted in time. For example, washing machines, cloth dryers, dishwashers, pool pumps, etc, can be shifted to different time-periods within their desired operation window for cost reduction. The BEMS model schedules the operation of such flexible loads in the desired window 
of operation specified by the user and total energy demand requirements of such loads need to be supplied to satisfy their operational constraints. These can be represented as:

$$
\begin{gathered}
x_{i, j}^{k} \leq 1 ; \quad \forall i \in \mathbf{T}_{\mathbf{t s}}, \forall j \in \mathbf{L}, \forall k \in \mathbf{N} \\
=0 ; \quad \text { Otherwise } \\
\sum_{i \in \mathbf{T}} x_{i, j}^{k} \mathbf{P}_{\mathbf{t s}} \Delta_{T}=E_{j}^{r e q} \quad \forall j \in \mathbf{L}
\end{gathered}
$$

here, $\mathbf{P}_{\mathbf{t s}} \in \mathbb{R}^{j \times k}$ represents the nominal power ratings in each power level for time-shiftable flexible loads.

Flexible loads can be further sub-categorized into interruptible and non-interruptible loads. For example, pool pumps are interruptible and time-shiftable as their operation can be shifted in time and their total operating time can be distributed into several short cycles. On the other hand, washing machine, cloth dryer, and dishwasher are time-shiftable flexible loads, however, they are non-interruptible loads. Typical operating cycles for such loads are shown in Fig. 1 and it is evident that economic losses may incur if such appliances are interrupted before completing their full operating cycle as they need to go over power extensive cycles. Therefore, a non-interrupted operation is desirable for such appliances and a penalty has been imposed for each interruption of non-interruptible loads, which is represented as follows:

$$
C_{i n t}=\sum_{j \in \mathbf{L}_{\mathbf{n i}}} \rho_{j}^{i n t}\left(\sum_{i \in \mathbf{T}} y_{i, j}^{k=1}-1\right)
$$

here, $k=1$ represents first power level in the operating cycles and penalty cost, $C_{\text {int }}$ will be 0 if $y_{i, j}^{k=1}=1$ only once in $T$. However, if $y_{i, j}^{k=1}=1$ for multiple values of $i \in T$ indicates that time-shiftable load $j$ is turned $\mathrm{ON}$ from OFF states more than once in a day, which represents an interrupted operation and penalty cost will be aggregated for all subsequent interruptions.

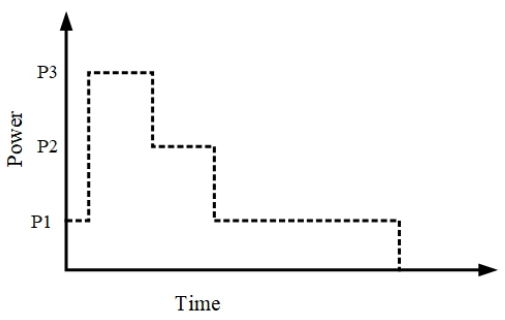

(a) Washing machine

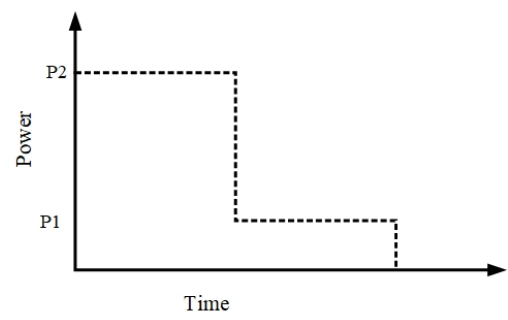

(b) Cloth dryer

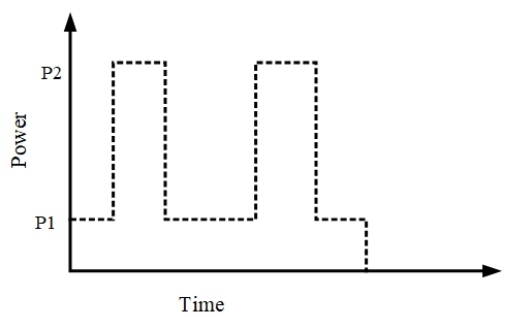

(c) Dishwasher

Figure 1: Typical operating cycles of time-shiftable and non-interruptible loads

\subsection{Storage model}

Typically, residential battery energy storage system (BESS) units are used to complement the intermittent renewable-based power generation from DG units such as rooftop photovoltaic (PV) systems. In typical PV-BESS systems, BESS units are charged with PV generated power during the day and this stored energy is used to supply higher residential load demand at the evening or night when grid supply is usually more expensive. On the other hand, the battery storage units of EV are charged when EV arrives home in the evening and continue charging up to maximum storage capacity, which is consumed by the EV for the daily commute in the following day. However, the proposed BEMS schedules the charge-discharge of both BESS and EV battery unit to offer maximum financial benefits for the prosumer while satisfying operational constraints and user preferences. The storage model also incorporates cycle-aging degradation of storage units to represents the actual financial benefits for the prosumer. The most notable stress factors on cycle-aging or lifetime degradation of the storage units include C-rate, over-current, non-optimal state of charge (SoC) \& depth of discharge (DoD), frequent charge-discharge cycles, over-charge \& over-discharge, temperature and 
humidity [27. The proposed BEMS charges and discharges the storage units at nominal power rating specified by the manufacturer thereby mitigating over-current situations. In addition to that, it also maintains the desired C-rate by considering manufacturer specified discharge power for optimal C-rate. On the other hand, external stress factors such as ambient temperature and humidity are not considered in the BEMS model as it is out of the scope of this paper. Therefore, effect of SoC, DoD and frequent charge-discharge cycles are only considered for cycle-aging degradation of the storage units which are discussed in following sections.

\subsubsection{State of charge (SoC)}

State of charge (SoC) represents the current storage level compared to maximum storage capacity. Therefore, SoC of a storage unit $j$ at time $i$ can be written as:

$$
\sigma_{i, j}=\sigma_{i-1, j}+\frac{\eta_{j}^{c}\left(x_{i, j}^{k=1} P^{c_{j}}\right)+\frac{1}{\eta_{j}^{d}}\left(x_{i, j}^{k=2} P_{j}^{d}\right)}{E_{j}^{m}} \Delta T \quad \forall i \in \mathbf{T}, j \in \mathbf{D}
$$

Over charging the storage units can lead to internal wear in battery cell. Therefore, over charging is prevented by limiting daily SoC within a maximum allowed threshold level as in Eq. (7), which represents maximum usable storage capacity.

$$
\sigma_{i, j} \leq \overline{S o C_{j}} \quad \forall i \in \mathbf{T}, j \in \mathbf{D}
$$

\subsubsection{Depth of discharge (DoD)}

Depth of discharge (DoD) of a storage unit represents the degree to which the unit is discharged compared to its maximum storage capacity. Therefore, DoD can be written in terms of SoC as:

$$
\delta_{i, j}=1-\sigma_{i, j} \quad \forall i \in \mathbf{T}, j \in \mathbf{D}
$$

Over discharging also leads to battery wear, hence, DoD is limited to a maximum threshold in the storage model, which represents minimum SoC value of the storage for optimal operation, and given as:

$$
\delta_{i, j} \leq \overline{D_{o D_{j}}} \quad \Rightarrow \quad \sigma_{i, j} \geq \underline{S o C_{j}} \quad \forall i \in \mathbf{T}, j \in \mathbf{D}
$$

If storage units are discharged at lower DoD, it reduces their life-cycle throughput. The marginal cycle aging degradation for each discharge cycle can be estimated as:

$$
\begin{array}{rlll}
\Phi_{i, j} & =\frac{\Delta \varphi_{i, j}}{\Delta \delta_{i, j}} \frac{\partial \delta_{i, j}}{\partial P_{i, j}^{d i s}} & & ; x_{i, j}^{k=2}=1 \quad \forall i \in \mathbf{T}, j \in \mathbf{D} \\
& =0 & & ; \text { otherwise }
\end{array}
$$

here, $x_{i, j}^{k=2}=1$ represents discharging of storage unit $j$ during $i$ and near quadratic DoD stress function for cycle degradation is used as shown in Eq. 11. The discharging power $P_{i, j}^{d i s}$ is estimated as in Eq. 12

$$
\begin{gathered}
\varphi(\delta)=(5.24 E-4) \delta^{2.03} \\
P_{i, j}^{d i s}=x_{i, j}^{k=2} P_{j}^{d} \quad \forall i \in \mathbf{T}, j \in \mathbf{D}
\end{gathered}
$$

Therefore, the cost for marginal degradation of discharge cycles can be calculated by factoring in battery replacement cost as follows:

$$
C_{D o D}^{d e g}=\sum_{j \in \mathbf{D}} C_{j}^{B} \sum_{i \in \mathbf{T}}\left(\Phi_{i, j} P_{i, j}^{d i s}\right)
$$




\subsubsection{Frequent charge-discharge}

Market driven operation of storage units can often lead to frequent and irregular charge-discharge cycles over a short span of time thereby reducing life-cycle expectancy of the storage units [27]. Therefore, a penalty cost is imposed for each subsequent charge-discharge cycles as:

$$
C_{c \leftrightarrow d}=\sum_{j \in \mathbf{D}} \rho_{j}^{c \leftrightarrow d}\left(\sum_{i \in \mathbf{T}}(\underbrace{y_{i, j}^{k=1} \& x_{i-1, j}^{k=2}}_{\text {discharge } \rightarrow \text { charge }} \| \underbrace{\& y_{i, j}^{k=2} \& x_{i-1, j}^{k=1}}_{\text {charge } \rightarrow \text { discharge }})\right.
$$

here, the first part of the boolean expression in Eq. (14) is TRUE when a storage unit $j$ switches to charging state from discharging state at time $i$ and the second part is TRUE in reverse situation. Hence, penalty cost is aggregated for such charge-discharge cycles over the operation window $\mathbf{T}$.

\subsection{Thermal model of the building with virtual energy storage systems (VESS)}

Thermal loads of a building include heat pumps (HP), heating ventilation and air conditioning (HVAC) systems, etc. Thermal loads generate heat to provide heating demand in winter and cooling demand in summer. The heat generated by thermal load can be written as:

$$
Q_{i}^{g e n}=\sum_{h \in \mathbf{H}} \kappa_{h} P_{h}^{t h} \quad \forall i \in \mathbf{T}
$$

where $P_{h}^{t h}$ is the power demand of the thermal load $h \in \mathbf{H}$.

Heat also generated internally from inhabitants, lightings and other electrical or mechanical appliances of the building. On the other hand, heat is exchanged between the building and external environment in thermal conduction at the boundary between two mediums as they are at different temperature. Heat exchange due to conduction can occur via the external surfaces of the building (walls and roof) and the building openings (windows and doors). Therefore, conduction heat exchanges can be written as:

$$
\begin{gathered}
Q_{i}^{c o n}=Q_{i}^{c s}+Q_{i}^{c w} \\
Q_{i}^{c s}=\sum_{p \in \mathbf{P}} \beta_{p} A_{p}\left(\theta_{i}^{\text {out }}-\theta_{i}\right) \\
Q_{i}^{c w}=\sum_{q \in \mathbf{Q}} \beta_{q} A_{q}\left(\theta_{i}^{\text {out }}-\theta_{i}\right)
\end{gathered}
$$

A fraction of the radiated heat is transmitted through the building openings (windows and doors) that depends on the solar radiation and heat transfer coefficients of the building openings. Some solar heat radiation is also absorbed by the surfaces of the building, which depends on the solar radiation, absorption

coefficient and heat resistance of the external surfaces. Therefore radiation heat exchanges can be written as:

$$
\begin{array}{r}
Q_{i}^{r a d}=Q_{i}^{r s}+Q_{i}^{r w} \\
Q_{i}^{r s}=\sum_{p \in \mathbf{P}} \alpha_{p} R_{p} A_{p} I_{i, p} \\
Q_{i}^{r w}=\sum_{q \in \mathbf{Q}} \tau_{q} A_{q} I_{i, q}
\end{array}
$$

Therefore, the power demand of the thermal loads to maintain the indoor temperature at the user specific temperature can be estimated as:

$$
Q_{i}^{g e n}=Q_{i}^{c o n}+Q_{i}^{r a d}-Q_{i}^{i n t} \quad \forall i
$$


Thermal loads are usually operated to provide a desired indoor thermal comfort level according to temperature set points specified by the user. However, a virtual energy storage system (VESS) is incorporated in this paper that utilizes the thermal mass of the building for a flexible operation of the thermal loads, whereby the building is considered as an isothermal mass of air volume and a flexible temperate range is used for indoor temperature instead of a fixed user specific temperature set-points. The use of thermal mass and flexible temperature range allows the thermal loads to precool or preheat the isothermal air volume during lower price periods (by prolonging operation time) and store the thermal energy to be used in higher price periods via the thermal mass of the air volume (by switching them off for certain periods) [33]. For such flexible operations, the thermal equilibrium equation for the building can be written as:

$$
\rho V C_{b} \frac{\Delta \theta_{i}}{\Delta_{T}}=Q_{i}^{i n t}+Q_{i}^{c o n}+Q_{i}^{r a d}+\sum_{h \in \mathbf{H}} \kappa_{h} P_{i, h}^{t h^{\prime}} \quad \forall i
$$

Therefore, it can be argued from Eq. 20 and Eq. 21) that the VESS is charging when $P_{i}^{t h^{\prime}}>P_{i}^{t h}$ and discharging when $P_{i}^{t h^{\prime}}<P_{i}^{t h}$. Therefore, the charging and discharging power for VESS can be estimated as:

$$
\begin{array}{llll}
P_{i}^{t h c} & =P_{i}^{t h^{\prime}}-P_{i}^{t h} & \forall P_{i}^{t h^{\prime}}>P_{i}^{t h} & \text { [charging] } \\
P_{i}^{t h d}=P_{i}^{t h}-P_{i}^{t h^{\prime}} & \forall P_{i}^{t h^{\prime}}<P_{i}^{t h} & \text { [discharging] }
\end{array}
$$

However, such flexible operation can often forces the indoor temperature to deviate further from the user specific temperature set-point thereby causing thermal discomfort for the consumer. Therefore, penalty is imposed for such thermal discomfort, which is modeled for the BEMS as follows:

$$
C_{d c m}=\rho^{d c m} \sum_{i \in \mathbf{T}}\left|\theta_{i}^{s e t}-\theta_{i}\right|
$$

\section{Day-ahead Scheduling and bidding model}

\subsection{Market framework}

In this paper, it is considered that the prosumer participates in a two-stage bi-directional market that includes a day-ahead (DA) wholesale energy market and a real-time (RT) local transactive market. In the DA market, the prosumer submits demand and supply quantity bids for each market interval of the 24 hours of the next day. Depending on the market arrangement, the tariff for demand and supply can be the same or different. The DA market is cleared before the gate closure of each day and tariffs are determined by the market coordinator. The market-clearing process is considered out of the scope of this paper, therefore, forecast of wholesale tariffs $(\hat{\pi})$ are considered for the DA market. Any imbalances from the DA market commitments are considered to be traded in the local RT market, which is coordinated by a local transactive aggregator. The full description of the local market is presented in 19 .

In this paper, the prosumer is considered to be a price taker i.e. it submits non-priced quantity bids of demand and supply for each market interval. The trading tariffs are considered to be determined by the market-clearing mechanism, which can be coordinated by a market coordinator (for the wholesale market) or transactive aggregator (for the local transactive market). As the market-clearing mechanisms are considered to be out of the scope, this paper only focuses on determining the optimal demand and supply quantity bids for the prosumer. The uncertainty in the RT market tariff is incorporated into the day-ahead scheduling model while determining DA energy bids. A maximum limit has been imposed on both demand and supply quantity bids for each market interval of DA and RT markets, which can represent the power transfer capacity of the residential LV networks.

\subsection{Uncertainty modeling}

The proposed BEMS schedules the building loads and DER units in day-ahead stages and determines the demand and supply quantity bids for the DA market to minimize energy cost. As the scheduling is determined in the day-ahead stage, different sources can contribute towards uncertainty including inflexible 
load demand, on-site DG generation, market prices (for both DA and RT markets), outdoor temperature, appliance usage, and EV availability. In this paper, it is considered that the prosumer provides the desired operation window for flexible appliance usages. In addition to that, tentative EV departure and arrival time is also considered to be known. Besides, the forecast prices for the DA wholesale energy market and outdoor temperature forecasts are considered to be fairly accurate. On the other hand, we consider inflexible load demand, on-site DG generation and RT market prices as the sources for uncertainty. The Monte Carlo Simulation (MCS)-based scenario generation and reduction technique of 31 is used in this paper to represent the uncertainties. Historical time-series data is used for forecasts, then the probability distribution function of the forecast errors are used to generate a large set of scenarios for all uncertain datasets. However, this makes the scheduling and bidding model a large-scale combinatorial optimization problem, therefore, the scenarios with comparatively lower probabilities are excluded and related scenarios in terms of statistical metrics are combined together according to 31 for reducing the problem size without losing the key statistical attributes of the historical datasets.

\subsection{Mathematical formulation}

The primary objective of the BEMS is to minimize the energy cost for the prosumer, therefore, the bidding problem can be formulated as a minimization problem to determine the optimal demand and supply quantity bids for the DA market that minimizes the electricity bills for the prosumer. However, a mere cost minimization model can also lead to non-optimal operation of building loads or DER units and can often affect user comfort. Therefore, the bidding model is formulated as a bi-level optimization problem that minimizes the energy cost in the upper level and a number of lower-level subproblems have been incorporated to ensure optimal operation for flexible building devices and maintain an optimal comfort level for the user. In addition to that, as the demand and supply quantity bids are optimized in the day-ahead stage, the uncertainties are incorporated by using a two-stage stochastic model for bidding optimization that includes a set of scenarios of power generation form DG units, non-flexible load demand, and RT market prices. The optimization model is represented as follows: 


$$
\begin{array}{ll}
\min & \sum_{i \in T}\left[\left(P_{i}^{+} \hat{\pi}_{i}^{+}-P_{i}^{-} \hat{\pi}_{i}^{-}\right) \Delta_{T}+\sum_{s \in S} \mathcal{P}_{s} \Delta P_{i, s}^{r t} \pi_{i, s}^{r t} \Delta_{T}\right] \\
\text { s.t. } & P_{i}^{+}-P_{i}^{-} \leq \hat{P}_{i}^{f l}+\sum_{j \in \mathbf{M}, k \in \mathbf{N}} x_{i, j}^{k} P_{i, j}^{k}+\sum_{h \in \mathbf{H}} P_{h, i}^{t h \prime}-\hat{P}_{i}^{g} \quad \forall i \\
& \Delta P_{i, s}^{r t}=\left(P_{i}^{+}-P_{i}^{-}\right)-\left(P_{i, s}^{f l}+\sum_{j \in \mathbf{M}, k \in \mathbf{N}} x_{i, j}^{k} P_{i, j}^{k}+\sum_{h \in \mathbf{H}} P_{h, i}^{t h \prime}-P_{i, s}^{g}\right) \quad \forall i, s \\
& \frac{P^{D A}}{P^{R T}} \leq\left(P_{i}^{+}-P_{i}^{-}\right) \leq \overline{P^{D A}} \\
& \underline{P_{i, s}^{r t}} \leq \overline{P^{R T}}
\end{array}
$$

Eqs. (1) and (2)

$\min C_{\text {int }}$

$$
\text { s.t. } x_{i, j}^{k}-x_{i-1, j}^{k}-y_{i, j}^{k}=0 \quad \forall i, \forall j \in \mathbf{L}_{\mathbf{n i}}, \forall k=1
$$

Eqs. (3) to (5)

$$
\begin{array}{cl}
\min & C_{D o D}^{\text {deg }}+C_{c \leftrightarrow d} \\
\text { s.t. } & x_{i, j}^{k}-x_{i-1, j}^{k}-y_{i, j}^{k}=0 \quad \forall i, \forall j \in \mathbf{D}, \forall k \in\{1,2\}
\end{array}
$$$$
\text { Eqs. (6) to 14] }
$$

$\min C_{d c m}$

$$
\text { s.t. } \theta_{i} \in[\underline{\theta}, \bar{\theta}]
$$

$$
\mathbf{x} \in\{0,1\}^{[i \times j \times k]} ; \mathbf{y} \in\{0,1\}^{[i \times j \times k]} ; \mathbf{P}^{\mathbf{t h} \prime} \in\left[0, \overline{\left.P^{t h \prime}\right]^{[j]}}\right.
$$

Here, the actual energy cost is formulated as the upper-level objective and other user inconveniences cost (thermal discomfort, interruption penalty, and storage degradation) are formulated as sub-problems in the lower-level as follows:

- Upper-level problem (Eqs. (24a) to (24f) and (24j)): The first part of the upper-level objective is the energy cost for the DA market, whereas the second part is the expected cost for real-time imbalances from the DA commitments over the set of uncertain scenarios. Here, $\hat{\mathcal{X}}$ indicates the forecast of variable $\mathcal{X}$, whereas $\mathcal{X}_{s}$ is the value of $\mathcal{X}$ in a particular scenario, $s \in S$, and $\mathcal{P}_{s}$ indicates the probability of that scenario. Therefore, the upper-level problem determines the demand and supply quantity bids for each market interval of the DA market that leads to minimum energy cost including all the scenarios of real-time realization for uncertainties. The demand bids represent the power demanded to supply building loads whereas the supply bids are the amount of power to be sold back to the grid. The power balance constraint in Eq. (24b) prevents over-bidding in the DA market, whereas the constraints in Eq. 24c ensures the supply-demand matching for the building over the set of scenarios in real-time. The constraints for the power-transfer capacities for the DA and RT markets are shown in Eqs. (24d) and $24 \mathrm{e}$, where $\mathcal{X}$ and $\overline{\mathcal{X}}$ indicates the minimum and maximum limits of $\mathcal{X}$ respectively with the superscript 'DA' and 'RT' indicating the DA and RT market respectively. The constraints for the binary decision variables are shown in Eq. (24f), and the bounds of the optimization variables are represented in Eq. 24j].

- Lower-level problem 1 (Eq. (24g)): The first sub-problem in the lower-level minimizes the interruption cost for non-interruptible flexible building loads subject to optimization constraints specified in Eq. 24g). The first constraint of Eq. (24g) ensures that the value of binary variable $y$ is 1 only when associated load switches its state from $\mathrm{OFF}$ to $\mathrm{ON}$, and it is 0 otherwise. 
- Lower-level problem 2 (Eq. $24 \mathrm{~h})$ ): The second lower-level subproblem minimizes the overall degradation cost of the storage units subject to their operational and optimization constraints for binary variables as indicated in Eq. $24 \mathrm{~h}$. Here, $y_{i, j}^{1}=1$ indicates the start of a charging cycle, and $y_{i, j}^{2}=1$ means that a discharging cycle has started at time, $i$ for storage unit, $j$.

- Lower-level problem 3 (Eq. (24i)): The third subproblem minimizes the thermal discomfort for flexible operation of thermal loads in the building subject to temperature constraints and operational constraints of the thermal loads.

Contrary to single-level (single-objective or multi-objective) formulations, proposed bi-level formulation allows minimizing the actual energy cost and determine the demand and supply quantity bids from the feasible set defined by the lower-level subproblems. In case of single-objective single-level formulation the different cost terms are added together, which is not feasible. On the other hand, multi-objective single-level formulation would consider all four objectives equally important, and provides a set of solutions (commonly known as Pareto font), which is feasible for all the constraints. However, such formulation does not reflect the actual objectives of the day-ahead scheduling and bidding problem of this paper.

\subsection{Solving algorithm}

The optimization model in Eq. (24) is a mixed-integer nonlinear bi-level problem, which is simplified in the following 3 steps to solve it using 'off the shelf' commercial software within a reasonable time.

\subsubsection{Step 1}

The nonlinearity from the optimization problem Eq. (24) is removed in this step. The nonlinearity is introduced into the problem due to the inclusion of constraints in Eq. (11) and (14). Linearization of these two constraints are discussed below:

- Linearizing Eq. (11) The nonlinear DoD stress function in Eq. (11) is replaced by equivalent piece-wise linear counterpart by including following constraints instead of Eq. (11).

$$
\begin{aligned}
& \delta_{i, j}^{a}+\delta_{i, j}^{b}=\delta_{i, j} \quad \forall i, \forall j \\
& \delta_{i, j}^{a} \leq x_{i, j}^{k=2} \quad \forall i, \forall j \\
& \delta_{i, j}^{b} \leq 1-x_{i, j}^{k=2} \quad \forall i, \forall j \\
& \sum_{d \in \mathcal{D}} \zeta_{i, j, d}=x_{i, j}^{k=2} \quad \forall i, \forall j \\
& \sum_{d \in \mathcal{D}} \delta_{i, j, d}^{p w}=\delta_{i, j}^{a} \quad \forall i, \forall j \\
& \zeta_{i, j, d} \frac{\delta_{d}^{p w} \leq \delta_{i, j, d}^{p w} \leq \zeta_{i, j, d} \overline{\delta_{d}^{p w}} \quad \forall i, \forall j, \forall d}{\varphi_{i, j}\left(\delta_{i, j}^{p w}\right)=\sum_{d \in \mathcal{D}}\left(a_{j, d} \delta_{i, j, d}^{p w}+b_{j, d} \zeta_{i, j, d}\right) \quad \forall i, \forall j}
\end{aligned}
$$

Here, $\delta^{a}$ and $\delta^{b}$ are auxiliary optimization variables. The constraints in Eqs. 25a to 25c indicate that the variable $\delta^{a}$ captures the value of DoD of a storage unit at any given time, $\delta_{i, j}$ if the storage, $j$ is discharging at that time, $i$ (as indicated by $x_{i, j}^{k=2}$ ). On the other hand, $\delta^{p w}$ and $\zeta$ are the optimization variables used to represent the active DoD segment in the piece-wise linearized degradation stress function of $\varphi$, where $d \in \mathcal{D}$ represents the linear line segments. When a storage unit is discharging with a specific DoD, then the corresponding linear line segment in the piece-wise linear model of $\varphi$ must be active, which is ensured by the constraint Eq. (25d) for binary variable, $\zeta$. When the active line segment is identified, then the identified DoD variable, $\delta^{p w}$ must fall within that active segment, which is ensured by constraints in Eqs. 25e and 25f. Here, $\delta_{d}^{p w}$ and $\overline{\delta_{d}^{p w}}$ are the initial and final value of the DoD levels in the piece-wise linearized line segment, $d$. Finally, Eq. (25g) is the linearized counterpart of the non-linear constraint in Eq. (11). 
- Linearizing Eq. (14) The nonlinearity in Eq. (14) is due to the boolean AND operation in the form

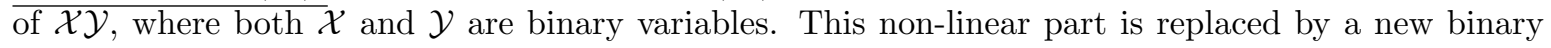
variable $\mathcal{Z}$ with following additional constraints:

$$
\begin{aligned}
& \mathcal{Z} \leq \mathcal{X} \\
& \mathcal{Z} \leq \mathcal{Y} \\
& \mathcal{Z} \geq \mathcal{X}+\mathcal{Y}-1 \\
& \mathcal{Z} \in\{0,1\}
\end{aligned}
$$

These constraints ensure that the value of $\mathcal{Z}$ is 1 only when both $\mathcal{X}$ and $\mathcal{Y}$ are 1 , which the boolean AND operation required for Eq. 14.

\subsubsection{Step 2}

Once the nonlinear constraints are replaced by equivalent linear constraints, the optimization problem becomes a bi-level mixed-integer linear problem. The traditional solutions of bi-level optimization problems include a reformulation of the problem into a single level problem where the lower level optimization problem is replaced by first-order Karush-Kuhn-Tucker (KKT) optimality conditions [34. In the proposed bidding optimization model, the variables controlled by a particular subproblem are exogenous to others in the lower-level, hence, the lower level sub-problems can be categorized as equilibrium problems of Nash type. Therefore, the same principle of bi-level decomposition applies here even though the bidding model contains multiple sub-problems at the lower level. Therefore, bi-level decomposition is applied to reformulate the problem as a Mathematical Program with Equilibrium Constraints (MPEC) by replacing the lower-level problems with their KKT conditions. The reformulated optimization problem can be written as:

$$
\begin{array}{ll}
\min & \text { Eq. 24a } \\
\text { s.t. } & \text { Eqs. 24b to 24f and 24j] }, \\
& \nabla_{\mathcal{X}_{p}} L_{p}\left(\mathcal{X}_{p}, \mu_{p, m}, \lambda_{p, n}\right)=0 \quad \forall p \in \mathcal{P}, \\
& \mu_{m, p} g_{m, p}\left(\mathcal{X}_{p}\right)=0 \quad \forall p \in \mathcal{P}, \forall m \in \mathcal{I}, \\
& \lambda_{n, p} h_{n, p}\left(\mathcal{X}_{p}\right)=0 \quad \forall p \in \mathcal{P}, \forall n \in \mathcal{J}, \\
& \mu_{m, p} \geq 0 \quad \forall p \in \mathcal{P}, \forall m \in \mathcal{I}
\end{array}
$$

where indices $p \in \mathcal{P}$ indicate the lower-level subproblems and $\mathcal{X}_{p}$ represents their variables. The equality and inequality constraints of lower-level optimization problems are represented by $h(\mathcal{X})$ and $g(\mathcal{X})$ respectively with $\mathcal{I}$ and $\mathcal{J}$ being their sets respectively. The KKT multipliers are represented by $\mu$ and $\Lambda$. The Lagrangian function is represented by $L_{p}\left(\mathcal{X}_{p}\right)$ for all the lower-level subproblems.

\subsubsection{Step 3}

However, the reformulation in step 2 introduces a new set of nonlinear constraints in Eqs. (27d) and (27e), which takes the form $C \mathcal{X}=0$. Fortuny-Amat McCarl linearization technique 34 is applied to replace such non-linear constraints in the reformulated problem by following linear constraints.

$$
\begin{array}{r}
0 \leq C \leq M u \\
0 \leq \mathcal{X} \leq M(1-u)
\end{array}
$$

where, $M$ is a large number and $u$ is a binary variable. For a large value of $M$ the constraints in Eq. (28) are the same as in Eqs. (27d) and 27e).

After the linearization and reformulation, the scheduling and bidding optimization problem becomes a single-level mixed-integer linear programming (MILP) problem, which can be solved reliably with available commercial software within feasible time. 


\section{Results and discussion}

The proposed methodology is evaluated via case studies for a residential prosumer in Sydney, Australia. The simulation is run for a summer and winter week. The input parameters and simulation set-up are discussed in Sections 4.1 to 4.3 then the simulation results and case studies are discussed in Section 4.4 . followed by a discussion in Section 4.5 .

\subsection{Description of the prosumer}

The prosumer building is a two-story Australian townhouse with a total floor area of $250 \mathrm{~m}^{2}$. The flexible loads of the building include a washing machine, a dishwasher and a pool pump. A heat pump is used in the building for space heating and cooling demand. The building is equipped with a rooftop solar PV system of $6 \mathrm{~kW}$ maximum capacity with a $14 \mathrm{kWh}$ Tesla Powerwall 2 BESS unit. The building is considered to be equipped with a BMW i3 electric vehicle with 94 Ah lithium-ion battery.

\subsubsection{Time-shiftable load parameters}

The nominal ratings of the flexible loads that can be shifted in time are listed in Table 1 No desired operation window is considered for the pool pump, rather it's operation is distributed in three periods of 2 hours and it is considered as non-interruptible during these 2 hours. In addition to that, for even distribution of the pool pump's operation, a minimum idle period of 6 hours is considered between subsequent operations. A penalty cost coefficient of $\$ 1 \mathrm{AUD} /$ interruption is used for non-interruptible flexible loads. Even though a single interruption would not yield such a high cost, a higher penalty cost ensures a non-interrupting operation.

Table 1: Nominal ratings and user preferences of time-shiftable flexible loads

\begin{tabular}{|c|c|c|c|}
\hline \multirow{2}{*}{ Device name } & \multicolumn{2}{|c|}{ Nominal ratings } & \multirow{2}{*}{ Desired operation window } \\
\hline & Power & Energy & \\
\hline Washing machine & $2.2 \mathrm{~kW}(\max )$ & $1.35 \mathrm{kWh} /$ load & $10 \mathrm{AM}-2 \mathrm{PM}$ \\
\hline Dishwasher & $2.0 \mathrm{~kW}(\max )$ & $1.50 \mathrm{kWh} / \mathrm{load}$ & $12 \mathrm{AM}-6 \mathrm{AM}$ \\
\hline Pool pump & $2.14 \mathrm{~kW}(9.3 \mathrm{~A})$ & $12.84 \mathrm{kWh} /$ day & - \\
\hline
\end{tabular}

\subsubsection{Storage parameters}

The input parameters of storage units (BESS and EV battery) used for the case studies are listed in Table 2. The same efficiency is considered for charging and discharging for both storage units with the manufacturer specified round-trip efficiency ( $89 \%$ for BESS and $92 \%$ for the EV battery). The nominal power demand for charging and discharging is considered the same, and it is considered as $3.6 \mathrm{~kW}$ for EV battery to prevent over-charge/discharge. The manufacturer recommended charge-discharge power is 3.3 $\mathrm{kW}$ for the BESS units for optimal C-rate [35]. Therefore, a continuous and fixed charging/discharging power of $3.3 \mathrm{~kW}$ is considered for the BESS unit even though its rated maximum power is $5 \mathrm{~kW}$. The usable energy of storage units is $18.8 \mathrm{kWh}$ for EV battery and $13.4 \mathrm{kWh}$ for BESS according to the manufacturer, which translates to $85 \%$ and $90 \%$ maximum DoD for EV battery and BESS respectively. Therefore, the minimum SoC limit of $15 \%$ (maximum DoD of $85 \%$ ) and $10 \%$ (maximum DoD of 90\%) are considered for them respectively. On the other hand, the maximum limit for SoC is considered $95 \%$ for both storage units. The penalty coefficients for frequent charge-discharge cycles are considered as 1.26çfor BESS and 1.06çfor EV battery (considering a loss-of-life of $\frac{1}{E^{m}}$-th of the energy-charged or discharged for each subsequent charge-discharge or discharge-charge cycle). These coefficients are estimated from manufacturer specified life cycles of 3200 for BESS and 4000 for EV battery. 
Table 2: Nominal ratings and parameters of the storage units

\begin{tabular}{lcc}
\hline Parameters & EV battery & BESS \\
\hline Rated Power (charge/discharge) & Level 2 charging $(3.3-6 \mathrm{~kW})$ & $5 \mathrm{~kW}(\max )$ \\
Maximum storage capacity & $22 \mathrm{kWh}(18.8 \mathrm{kWh}$ usable) & $14 \mathrm{kWh}(13.4 \mathrm{kWh}$ usable) \\
Charging and discharging efficiencies $\left(\eta^{c}, \eta^{d}\right)$ & $96 \%$ & $94.5 \%$ \\
Charging and discharging power $\left(P^{c}, P^{d}\right)$ & $3.6 \mathrm{~kW}$ & $3.3 \mathrm{~kW}$ \\
SoC limits $(\underline{S o C}, \overline{S o C})$ & $15 \%, 95 \%$ & $10 \%, 95 \%$ \\
Replacement cost $(\mathrm{AUD})$ & $\$ 13000$ & $\$ 9000$ \\
\hline
\end{tabular}

\subsubsection{Thermal load parameters}

The prosumer building is considered to be equipped with a reverse-cycle heat pump for heating and cooling demand. A thermal model of the building is proposed in this paper with a VESS for the flexible operation of the heat pump. The input parameters of the heat pump and building parameters used in the case studies are presented in Tables 3 and 4 . The forecast outdoor temperatures used for the operation of the heat-pump are shown in Fig. 2 for the summer and winter weeks.

Table 3: heat pump parameters

\begin{tabular}{lcc}
\hline Parameters & Summer & Winter \\
\hline Thermostat set-point & $21^{\circ} \mathrm{C}$ & $25^{\circ} \mathrm{C}$ \\
Temperature range & $19^{\circ} \mathrm{C}-23^{\circ} \mathrm{C}$ & $23^{\circ} \mathrm{C}-27^{\circ} \mathrm{C}$ \\
CoP of heat pump & \multicolumn{2}{c}{2.5} \\
$\rho^{d c m}\left(c /{ }^{\circ} \mathrm{C}\right)$ & \multicolumn{2}{c}{5} \\
\hline
\end{tabular}

Table 4: Building parameters

\begin{tabular}{|c|c|c|c|c|c|c|c|c|c|}
\hline \multirow{2}{*}{$\begin{array}{c}\rho \\
\left(k g / m^{3}\right)\end{array}$} & \multirow{2}{*}{$\begin{array}{c}V \\
\left(m^{3}\right)\end{array}$} & \multirow{2}{*}{$\begin{array}{c}C_{b} \\
\left(J / k g^{\circ} C\right)\end{array}$} & \multicolumn{2}{|r|}{ Area $\left(m^{2}\right)$} & \multicolumn{2}{|c|}{$\beta\left(\mathrm{W} / \mathrm{m}^{2} \mathrm{~K}\right)$} & \multirow{2}{*}{$\begin{array}{c}\mathrm{R}_{s} \\
\left(\mathrm{~m}^{2} \mathrm{~K} / \mathrm{W}\right)\end{array}$} & \multirow[t]{2}{*}{$\alpha_{s}$} & \multirow[t]{2}{*}{$\tau_{w}$} \\
\hline & & & surface & windows \& doors & surface & Windows \& doors & & & \\
\hline 1.2 & 625 & 1000 & 420 & 80 & 1.7 & 2.75 & 3.2 & 0.4 & 0.08 \\
\hline
\end{tabular}

\subsubsection{Fixed-load and solar $P V$ forecasts and scenarios}

One year's time-series meter-measurement data for fixed-load consumptions and PV generation of the prosumer is used to forecast the fixed-load demand and PV generation for a summer and winter week. The forecasting is done using Matlab Artificial Neural Network (ANN) toolbox. Based on the distribution of forecast errors, the scenarios are generated and reduced to 20 scenarios according to the methodology presented in 31. The input data used for forecasting fixed-load demand are the time-series datasets for fixed-load demand and outdoor temperature, whereas annual time-series datasets of PV generation and temperature are used as input for forecasting PV generation. The forecast profiles and scenarios of the fixed-load demand and PV generation used for simulations are shown in Figs. 3 and 4 along with their actual profiles for both summer and winter week.

\subsection{Market tariff}

Based on the historical wholesale market data, the tariff for the DA energy market is forecast for the summer and winter week according to the methodology presented in [36, which is shown in Fig. 5a. The DA demand and supply tariff are considered the same (i.e. $\pi^{+}=\pi^{-}$). Determining the RT market tariff for the local RT market would require a complete simulation of the local TE market clearing mechanism and 


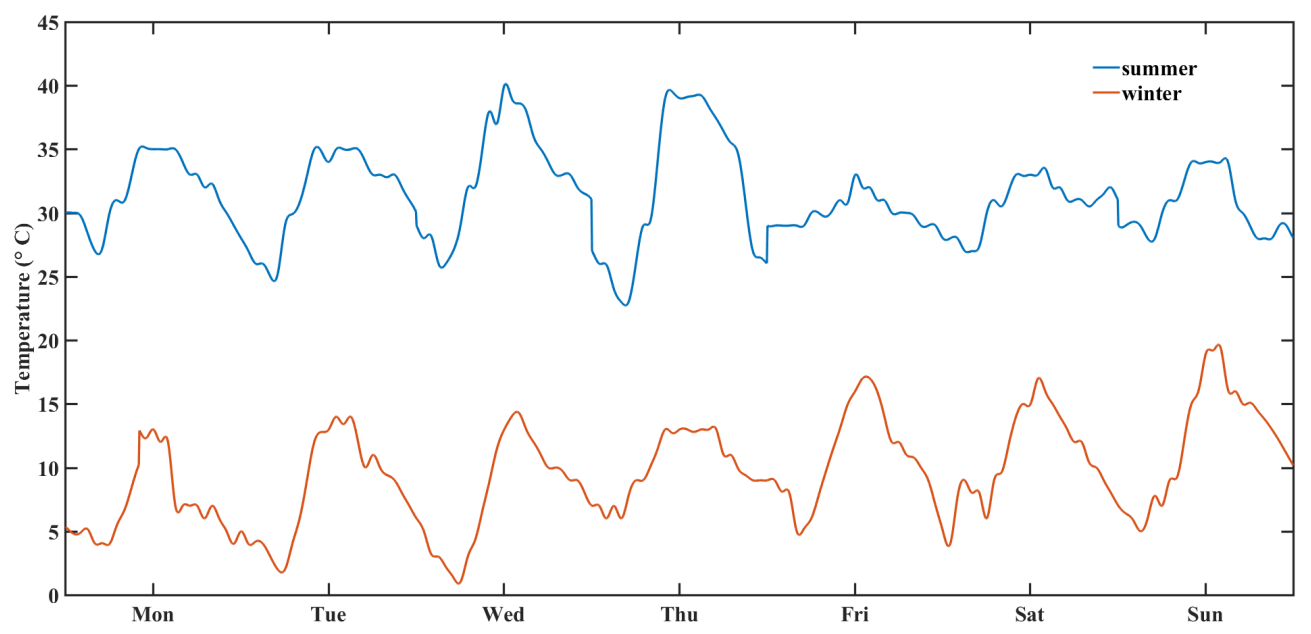

Figure 2: Outdoor temperature for the summer and winter week

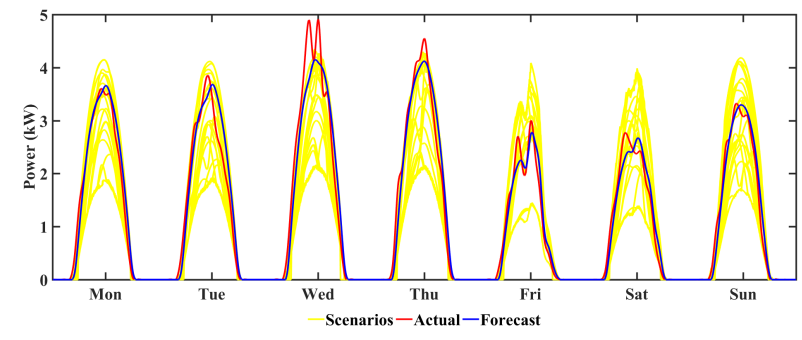

(a) summer

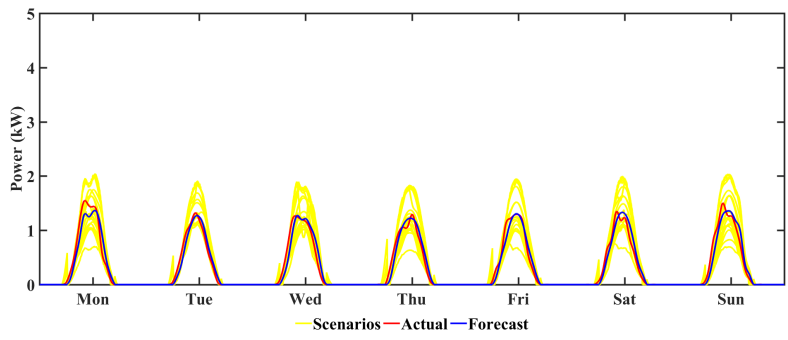

(b) winter

Figure 3: PV generation for the summer and winter week

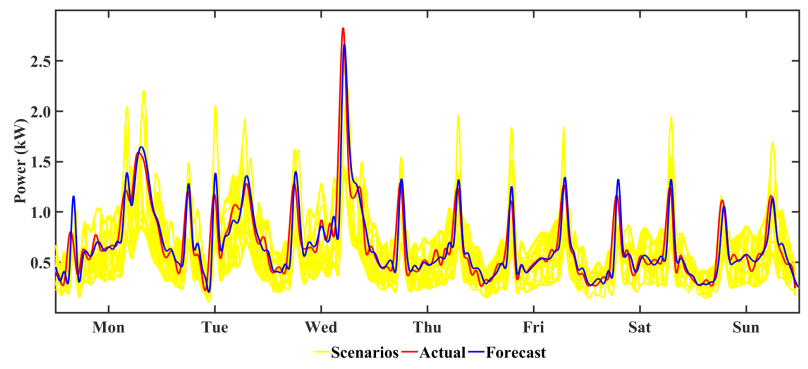

(a) summer

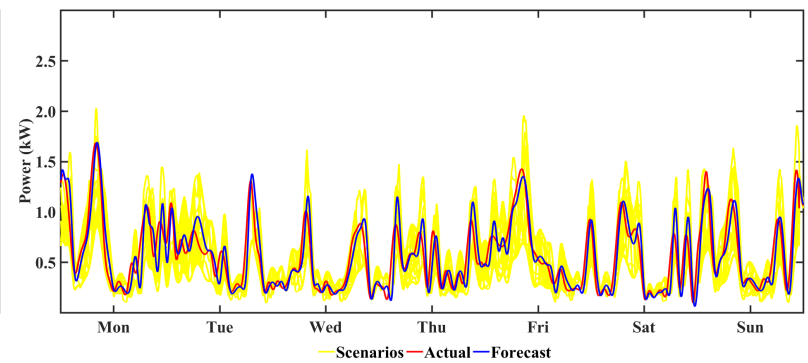

(b) winter

Figure 4: Fixed-load demand for the summer and winter week 
power flow studies of the local LV network, which is considered out of the scope of this paper. The local market presented in [19] is simulated for one year and based on that the scenarios for RT market tariffs are generated according to 31 , which are shown in Figs. $5 \mathrm{~b}$ and $5 \mathrm{c}$

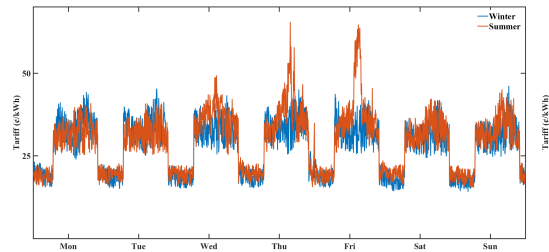

(a) Wholesale tariff

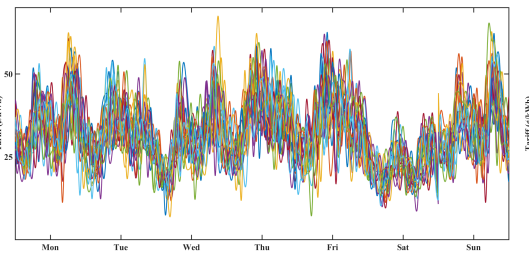

(b) Local market tariff (summer)

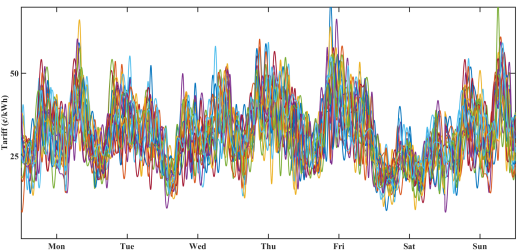

(c) Local market tariff (winter)

Figure 5: Market tariffs for the summer and winter week

\subsection{Optimization set-up}

For the piecewise linearization of step 1 in Section 3.4, 10 linear line segments are considered, which gives a root-mean-squared error (RMSE) of less than $0.009 \%$. For the step 3 in Section 3.4 , the value of $M$ is considered as $10^{5}$. The simulation periods of 5 minutes are considered in line with the Australian wholesale market bidding period. The case studies are run one day at a time for the summer and winter week data, and the SoC of the storage units found at the end of the day is considered as the initial SoC for the next day. The storage degradation is considered for discharging only, assuming that the storage units go through the same number of charging cycles as the discharging cycles. Therefore, the final SoC at the end of the week is considered the same as the initial SoC at the beginning of the week. The reformulated MILP optimization problem is formulated in General Algebraic Modeling System (GAMS) language and solved using CPLEX solver 37 in a computer with Intel Core i7, 3.4 GHz processor and 16 GB of RAM. The absolute and relative optimality gap is set at 0 for all simulation studies, and the computation time is found to be within 3 seconds for all simulation studies.

\subsection{Simulation and results analysis}

Based on the simulation parameters discussed in the previous sections, the scheduling and bidding optimization model is simulated for a summer and winter week. The demand and supply quantity bids for the summer and winter weeks are discussed in Section 4.4.1, followed by the discussion on the results in terms of device scheduling in Section 4.4.2. The effect of scheduling and bidding strategies on the cost of the prosumer is discussed in Section 4.4.3, where a few 'state-of-the-art' strategies are compared with the proposed methodology to evaluate its performance in terms of cost savings for the prosumer.

\subsubsection{DA demand and supply quantity bids}

The bidding optimization model determines energy bids in day-ahead stages to minimize electricity costs subject to minimum storage degradation, thermal discomfort, and user inconveniences. Contrary to deterministic approaches, the proposed stochastic method weighs the expected cost for real-time imbalance trading for the uncertain sets of fixed-load demand, PV generation, and RT market tariff scenarios. The demand and supply quantity bids for the first day of both summer and winter weeks are shown in Fig. 6 . along with the RT imbalance bids for all the scenarios. The forecasts of PV generation and DA tariffs for that day are also shown in the figure, along with their scenarios. The negative bids in the figure indicate supply bids. The demand bids are found to be comparatively higher when the market tariff is lower to minimize energy consumption cost for the prosumer. On the other hand, comparatively higher supply bids are noticed during the peak tariff periods or when PV generation is higher. Due to the inclusion of the stochastic term in the upper-level objective, the proposed method submits conservative DA demand and supply quantity bids when uncertainty is higher, especially during the mid-day due to higher uncertainties in PV generation. The effect of scheduling and bidding strategies on energy bids and costs are further discussed in Section 4.4.3. 


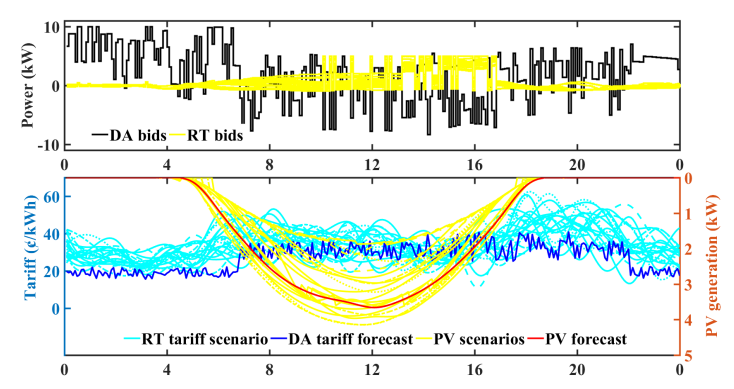

(a) Monday (summer)

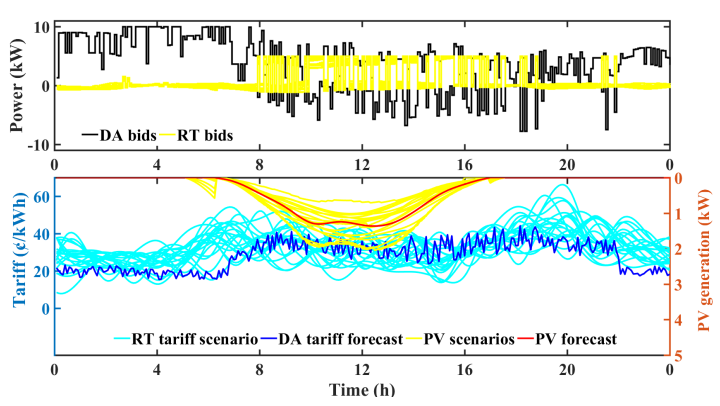

(b) Monday (winter)

Figure 6: DA quantity bids for demand and supply

\subsubsection{Device scheduling}

The proposed method schedules the building devices according to user-specified preferences and operational constraints of the devices explained in Section 2. The schedules of building devices for some selected days are explained in the following sections.

\subsubsection{Time-shiftable flexible loads}

The schedules of time-shiftable flexible loads for two subsequent days are shown in Fig. 7 It can be seen that the BEMS schedules all three time-shiftable loads within their desired operational windows specified by the user and according to the constraints discussed in Section 4.1. In addition to that, the sequences of power-level for these loads are also ensured, and the minimum duration of 6 hours is maintained between subsequent operations of pool-pump.

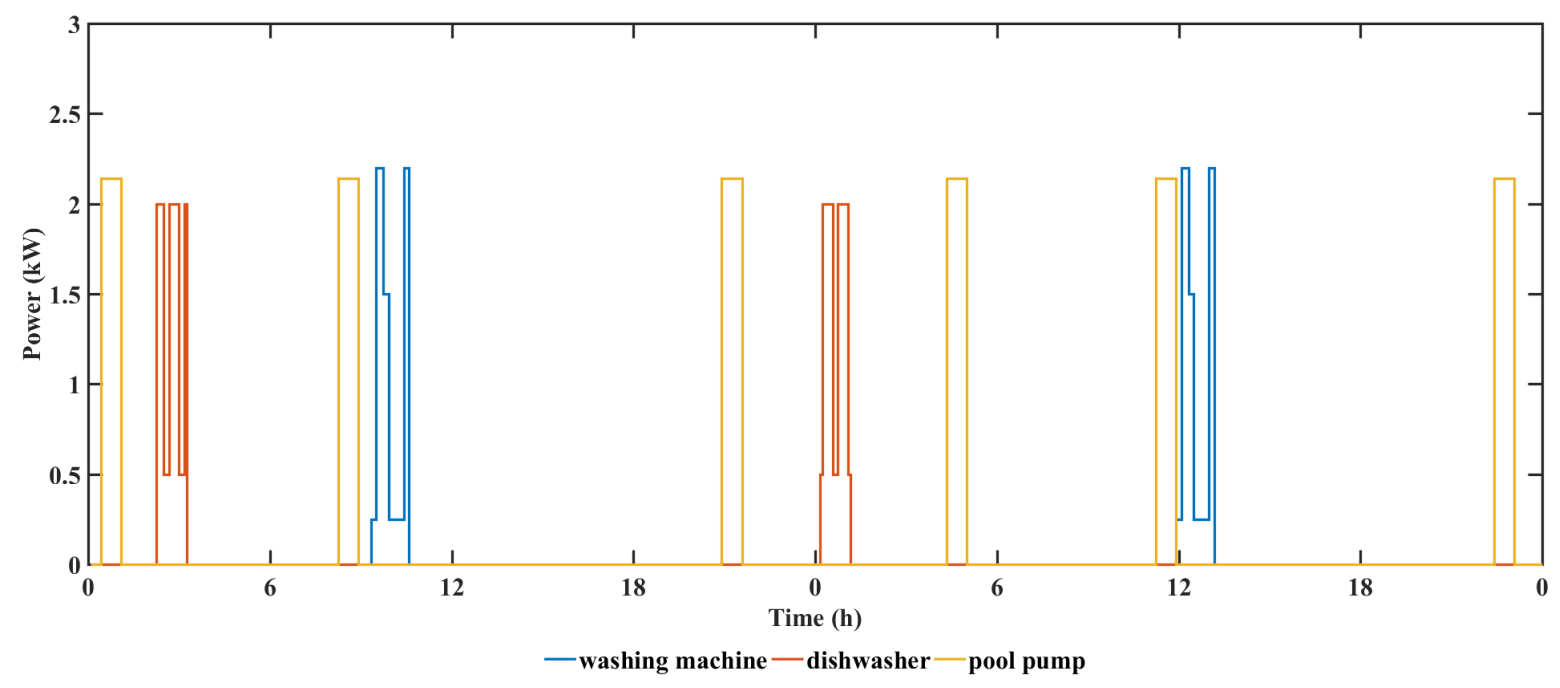

Figure 7: Schedule of time-shiftable flexible loads for 2 days

\subsubsection{Storage units}

Based on these input parameters discussed in Section 4.1, the BEMS schedules the charging and discharging of storage units that lead to minimum energy cost subject to minimum degradation of storage units. The 
schedules for the BESS unit is shown in Fig. 8 for two subsequent days. It can be seen that the BESS unit is mostly charged during higher PV generation or when electricity is cheaper. Besides, the SoC constraints of the BESS unit are also satisfied, and subsequent charge-discharge or discharge-charge cycles are minimized to reduce the frequent charge-discharge penalty cost as discussed in Section 2.3 .

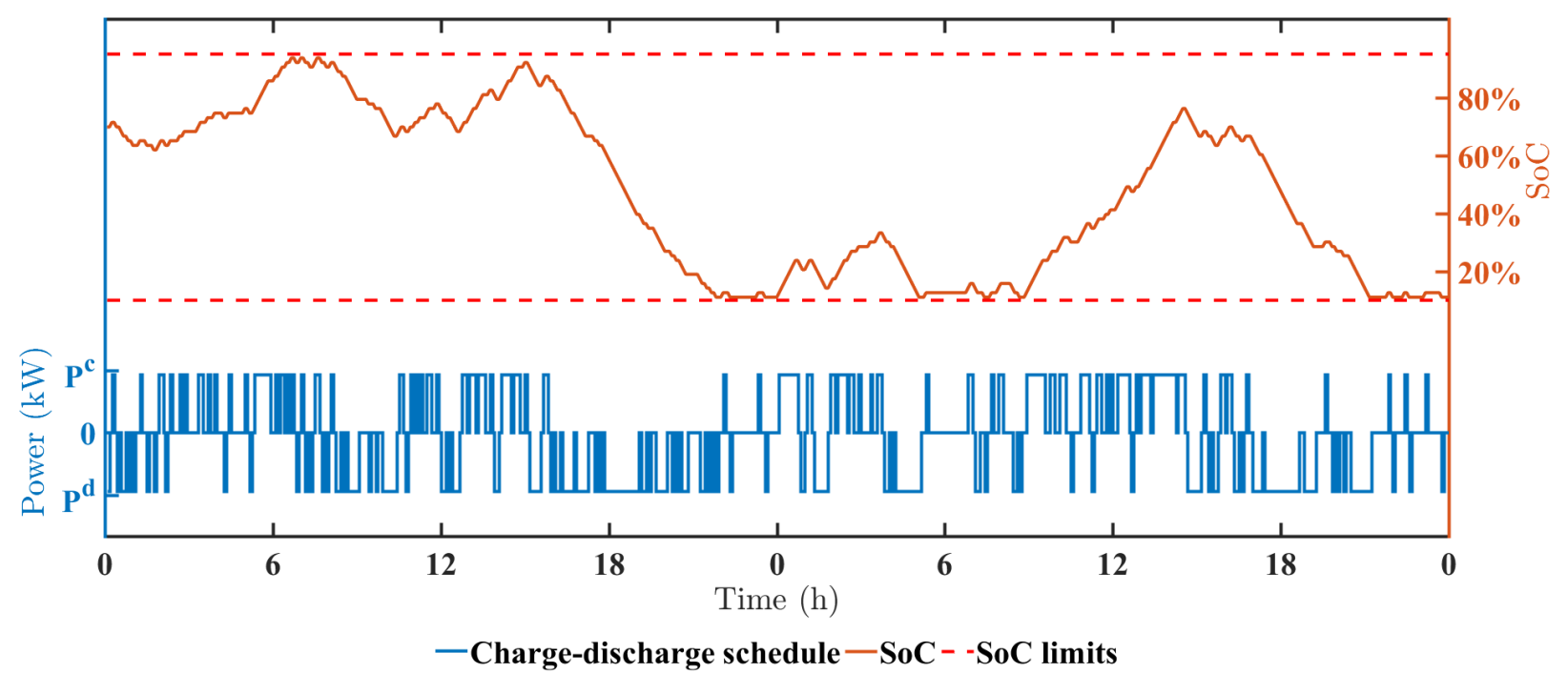

Figure 8: Schedule of BESS for 2 days

The EV availability, SoC level at arrival and the energy consumption for daily commute are forecasted form the Australian "Smart Grid Smart City" EV trial data 38, which provides EV trip data of 20 EVs for two years. For the EV battery, it is also considered that the user wants the EV battery to be charged at least a certain level before leaving home in the morning. The SoC requirements in departure are arbitrarily considered between $70 \%$ - 90\% (excepts for the weekend) in line with the EV travel pattern in 38. The SoC levels of the EV for a week are shown in Fig. 9. The periods when EV is away from home are highlighted in the figure along with the arrival time. It can be seen that the scheduling model satisfies all the SoC constraints.

\subsubsection{Thermal loads}

The BEMS schedules the operation of the heat pump to maintain the indoor temperature within a flexible range subject to minimum deviation from thermostat set-point temperatures (as the bidding model penalizes such deviations) and determines schedules of heat pump that corresponds to minimum thermal discomfort for the user. The schedules of heat pump for a simulated winter day is shown in Fig. 10, with associated indoor and outdoor temperature profiles. The heat pump power demand without VESS $\left(P^{t h}\right)$ is also indicated in the figure along with the demand with $\operatorname{VESS}\left(P^{t h^{\prime}}\right)$.

It can be seen that the heat pump is operated to maintain indoor temperature stays within user-specified limits throughout the day. The thermal model of the BEMS incorporates a VESS that utilizes the thermal mass of the building to economically schedule the heat pump subject to minimum thermal discomfort for the building inhabitants. The use of thermal mass and flexible temperature range allow the BEMS to achieve a heat pump energy demand reduction of $15-22 \%$ in summer and $10-12 \%$ in winter. Due to the use of the thermal mass of the building, it can be seen from Fig. 10 that there are few instances when the heat pump is not consuming any energy, still, the indoor temperature increases as the VESS is discharging its stored energy during these instances. 


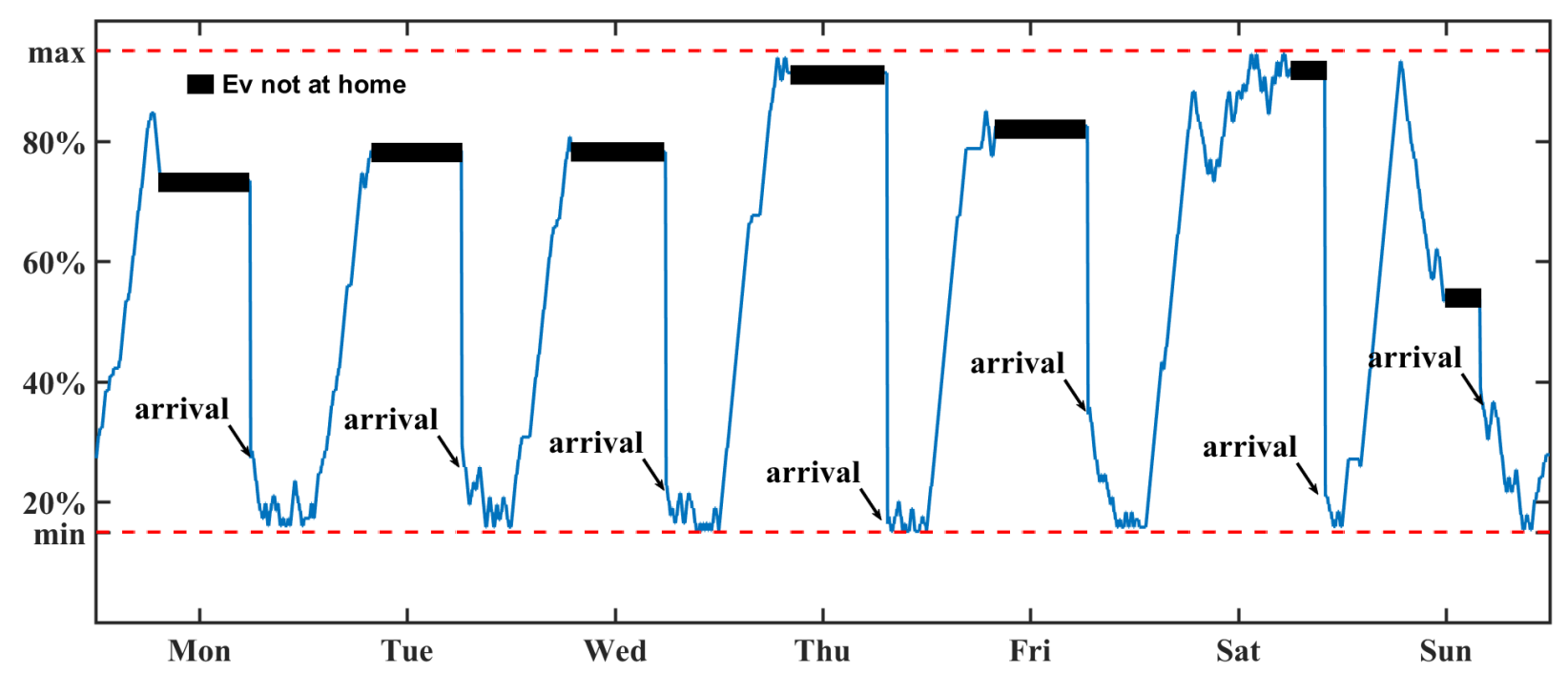

Figure 9: EV SoC level for a week

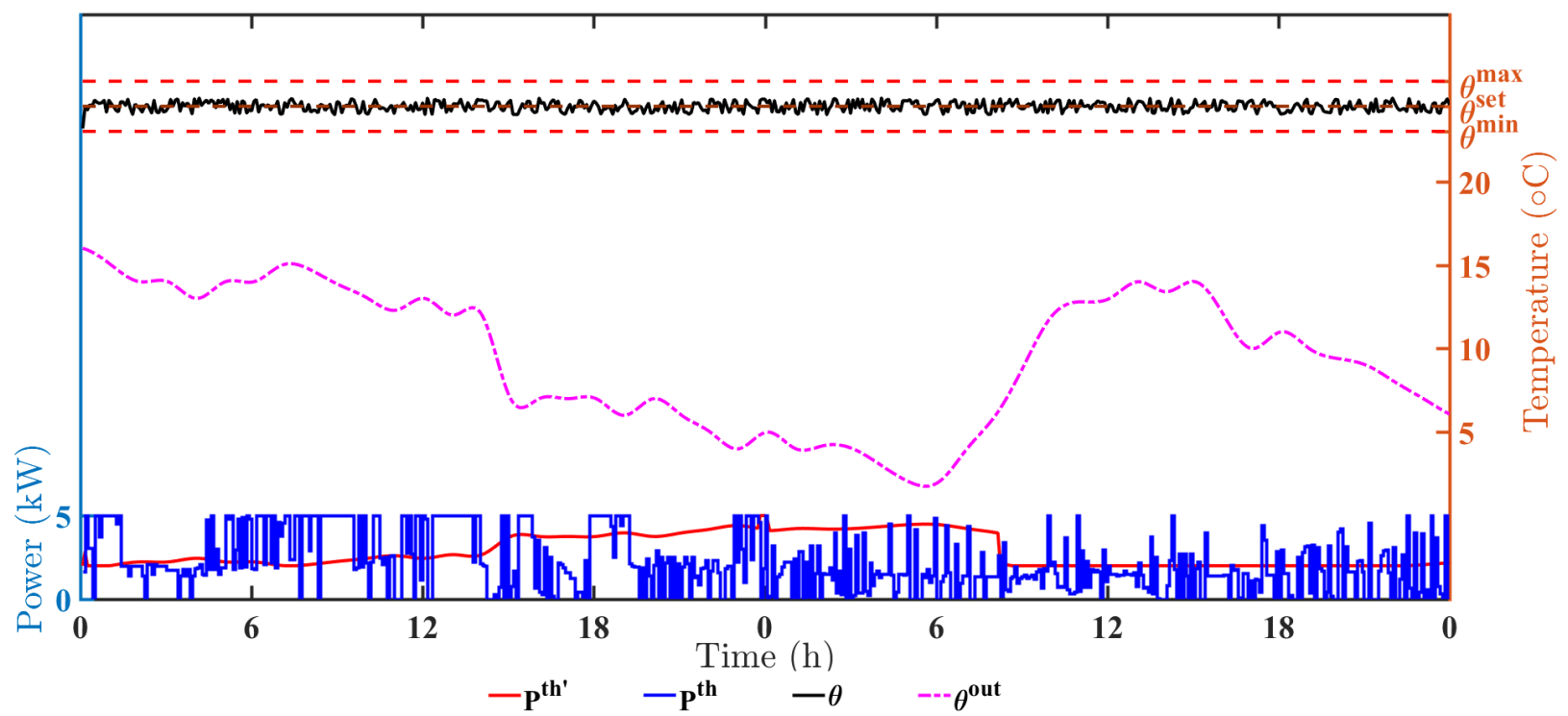

Figure 10: Schedule of heat pump for a winter day 
As discussed in Section 2.4 the VESS is charging when $P_{i}^{t h^{\prime}}>P_{i}^{t h}$ and discharging when $P_{i}^{t h^{\prime}}<P_{i}^{t h}$. It can be seen in Fig. 10 that, VESS mostly discharges during peak tariff periods to reduce overall demand bids for the prosumer. It also allows the BEMS to pre-heat or pre-cool the building when electricity is cheaper or when DERs are available using the VESS, which can be later used to provide thermal demand during peak-tariff periods by discharging VESS.

However, as the primary objective is cost minimization, the indoor temperature in Fig. 10 is found to have deviated from set-point temperatures when cost reduction for such flexible operation is higher compared to discomfort cost. The heat-pump power demand of the proposed methodology is compared with the estimated power demand of the building simulated in EnergyPlus. EnergyPlus is a building energy simulation program capable of calculating electric power demand for space heating and cooling to maintain a specific indoor temperature [33, 39. It is widely used as a building energy benchmarking tool 33, 40, 41. A residential building is simulated in the EnergyPlus software with the parameters specified in Tables 3 and 4 and the power demand for space heating and cooling are estimated to maintain the same indoor temperature profiles found for the simulated summer and winter week. The HP power demand of the proposed BEMS is then compared with the EnergyPlus-simulated power demand and associated percentage error in estimated for all simulation cases of summer and winter. The percentage error is found to be less than $4 \%$ for all simulation instances with a mean percentage error found to be $1.83 \%$. Therefore, it can be argued that the proposed methodology is capable of estimating the electric power demand of the heat pump for space heating and cooling with acceptable accuracy.

\subsubsection{Case studies}

Case studies have been conducted to analyze the total cost for the prosumer for different scheduling strategies, and the proposed two-stage stochastic bi-level optimization-based scheduling and bidding strategy is compared with these strategies to evaluate its effectiveness in terms of actual cost savings for the prosumers.

\subsubsection{Description of strategies}

Four strategies are considered to determine the day-ahead scheduling and DA demand-supply quantity bids for the prosumer with the same input data and parameters discussed in Sections 4.1 to 4.2 . The strategies are as follows:

1. Strategy 1 - Inflexible scheduling

The inflexible strategy represents the scenario when building appliances are operated without any flexibility. For this strategy, it is considered that the washing machine and dishwasher are operated during the evening on weekdays, and they are operated at night on weekends. The prosumer usually runs the pool pumps overnight for 6 straight hours, which is considered in the inflexible strategy. For this strategy, it is considered that the EV battery starts charging upon arrival until it is up to the same level as the proposed method. On the other hand, the BESS unit is considered to be charged with power generated from the PV system to be used during peak tariff periods (i.e. evenings). The excess energy injection to the grid is not considered in the scheduling strategy. The heat pump is operated to maintain the indoor temperature the same as the thermostat set-points. The power demand of the heat pump for the base case is estimated according to Eq. 20). In conclusion, the inflexible strategy indicates the non-optimal worst-case scenario of scheduling and place most demand quantity bids during the evening (after utilizing the storage BESS energy), when electricity is expensive.

2. Strategy 2 - Deterministic bi-level optimization

This strategy is the deterministic day-ahead schedule. Therefore, the second part of the upper-level objective in Eq. 24a is removed along with the uncertain scenarios. As a result, it minimizes the energy cost based on forecast information. The lower-level subproblems are kept the same as the 
proposed method. The optimization model for this strategy can be expressed as:

$$
\begin{array}{ll}
\min & \sum_{i \in T}\left(P_{i}^{+} \hat{\pi}_{i}^{+}-P_{i}^{-} \hat{\pi}_{i}^{-}\right) \Delta_{T} \\
\text { s.t. } & P_{i}^{+}-P_{i}^{-}=\hat{P}_{i}^{f l}+\sum_{j \in \mathbf{M}, k \in \mathbf{N}} x_{i, j}^{k} P_{i, j}^{k}+\sum_{h \in \mathbf{H}} \hat{P}_{h, i}^{t h \prime}-\hat{P}_{i}^{g} \quad \forall i, \\
& \text { Eqs. 24d and 24f to 24j }
\end{array}
$$

This scheduling problem is formulated as a bi-level deterministic optimization, which is reformulated as a MILP problem according to the methodology discussed in Section 3.4 and solved similarly to the proposed method with the same input parameters and optimization setup.

3. Strategy 3 - Single-level two-stage stochastic optimization minimizing energy cost only

In the strategy, the uncertainty is considered, however, the scheduling and bidding model is formulated as an optimization problem that only minimizes the energy cost. As the storage degradation cost, thermal discomfort cost and interruption cost of flexible loads are not considered in this strategy, the scheduling and bidding problem for this case can be formulated as a single-level two-stage stochastic problem to minimize energy cost considering the expected cost for uncertainties. Therefore, the optimization problem for this strategy can be written as:

$$
\begin{array}{ll}
\min & \sum_{i \in T}\left[\left(P_{i}^{+} \hat{\pi}_{i}^{+}-P_{i}^{-} \hat{\pi}_{i}^{-}\right) \Delta_{T}+\sum_{s \in S} \mathcal{P}_{s} \Delta P_{i, s}^{r t} \pi_{i, s}^{r t} \Delta_{T}\right] \\
\text { s.t. } & \text { Eqs. (3) to (14), Eqs. (16) to 23), Eqs. 24b to (24f) and 24j) }
\end{array}
$$

This non-linear problem is linearized according to the first step in Section 3.4, and then solved in GAMS using the same solver and optimality conditions as the proposed method.

4. Strategy 4 - Single-level multi-objective two-stage stochastic optimization

In the strategy, scheduling and bidding model is formulated as a single-level optimization problem that minimizes the energy cost, storage degradation cost, thermal discomfort cost and the interruption cost for the flexible loads. As there are multiple objectives to be minimized simultaneously, it can be formulated as a multi-objective model as follows:

$$
\begin{aligned}
& \min \left[\begin{array}{llll}
F_{1} & F_{2} & F_{3} & F_{4}
\end{array}\right] \\
& \text { s.t. } \quad F_{1}=\sum_{i \in T}\left[\left(P_{i}^{+} \hat{\pi}_{i}^{+}-P_{i}^{-} \hat{\pi}_{i}^{-}\right) \Delta_{T}+\sum_{s \in S} \mathcal{P}_{s} \Delta P_{i, s}^{r t} \pi_{i, s}^{r t} \Delta_{T}\right] \text {, } \\
& F_{2}=C_{D o D}^{d e g}+C_{c \leftrightarrow d}, \\
& F_{3}=C_{d c m} \\
& F_{4}=C_{\text {int }} \text {, } \\
& \text { Eqs. (3) to 14, Eqs. 16) to 23, Eqs. 24b to 24j }
\end{aligned}
$$

As the expected cost of the uncertainties is also included in the objectives, the formulated problem is a stochastic multi-objective minimization problem. The nonlinearity in the model is replaced by equivalent linear counterparts according to step 1 in Section 3.4. Traditional solution approaches of such multi-objective optimization problems include the weighted-sum method, epsilon-constraint method, weighted metric method, etc. [28, 42, that provide a set of solutions, called Pareto optimal solution sets. The Pareto optimal set is a set of solutions that do not dominate each other, and different points in the set indicate different trade-off combinations between the objectives. Therefore, moving from one point in the Pareto set to other(s) would mean sacrificing one objective(s) in favor of other(s). To solve this multi-objective problem, the objectives are first normalized by solving them 
Table 5: Cumulative results of scheduling and bidding strategies for summer week

\begin{tabular}{lccccc}
\hline & \multicolumn{5}{c}{ Scheduling and bidding strategy } \\
\cline { 2 - 6 } & Strategy 1 & Strategy 2 & Strategy 3 & Strategy 4 & Proposed method \\
\hline DA demand bids $(\mathrm{kWh})$ & 592.93 & 605.88 & 608.65 & 539.27 & 512.46 \\
DA supply bids $(\mathrm{kWh})$ & - & 120.46 & 396.46 & 273.47 & 260.40 \\
Total DA energy cost $(\$)$ & 148.43 & 93.61 & -8.48 & 14.27 & 11.50 \\
Expected RT bids $(\mathrm{kWh})$ & - & & 207.90 & 214.30 & 209.26 \\
Expected imbalance cost $(\$)$ & - & 1.26 & 60.75 & 62.67 & 61.11 \\
Net energy cost $(\$)$ & 148.43 & 92.35 & 52.27 & 76.94 & 72.60 \\
Storage degradation cost $(\$)$ & 6.23 & 14.32 & 64.24 & 24.64 & 12.29 \\
Thermal discomfort cost $(\$)$ & 0 & 9.87 & 31.95 & 6.43 & 8.63 \\
Interruption cost $(\$)$ & 0 & 0 & 5 & 0 & 0 \\
\hline
\end{tabular}

individually as a maximization and minimization problem. Then, the weighted-sum method is applied to get the solutions of the equivalent single-objective problem. The objective function for the equivalent single-objective of Eq. (31a) can be written as:

$$
\min \sum_{a} w_{a, b} \frac{F_{a, b}-F_{a, b}^{\min }}{F_{a}^{\max }-F_{a}^{\min }} \quad \forall b
$$

where, $a \in\{1,2,3,4\}$ indicates the objective number and $F_{a}^{\max }$ and $F_{a}^{\text {min }}$ are the maximum and minimum value of individual objectives. The weights of the objectives are indicated by $w_{a, b}$ for a combination, $b$. The combinations for weights can be estimated by varying the weights of each objective between 0 and 1 . However, only the combinations that sums to total weight of 1 are valid (i.e. $\sum_{a} w_{a, b}=1 \quad \forall b$ ). There can be thousands of combinations with a smaller value of step size (for example, for 0.1 step-size there can be as many as 10000 possible combinations, out of which 72 satisfy the condition, therefore, $b=\{1,2,3, \ldots, 72\})$. This makes the problem a large-scale combinatorial optimization problem. The scope of this case study is to compare the effect of such multi-objective scheduling on the cost and compare it with the proposed method. Therefore, instead of comparing all the combinations, the multi-objective model is solved for equal weights (i.e. $b=\{1\}$ and $w_{a}=$ $0.25 \forall a$ in Eq. (32). The input parameters and optimization setup are kept the same as the proposed methodology.

\subsubsection{Effect of scheduling strategies on cost}

The cumulative results of the scheduling and bidding strategies are listed in Tables 5 and 6 for the summer and winter week respectively. The total energy consumption and energy cost for the winter week are found to be comparatively higher than that of summer due to lower PV generation and higher heat pump demand in winter. In this section, the results of the strategies 1 to 4 are compared with the proposed method in terms of energy cost, storage degradation cost, load interruption cost and thermal discomfort cost.

1. Strategy 1 vs proposed method

As discussed in Section 4.4.3.1 the first strategy is an inflexible strategy, where the V2B and V2G functionalities of EV are not considered. Besides, the PV generated power is considered to be stored in the BESS and used in the evening. In addition to that, the energy selling to the grid is also not considered in this strategy. Moreover, the cost-saving flexibilities of the flexible building loads and DER units are also not considered in this case. For example, the heat pump is considered to be operated for maintaining a fixed indoor temperature. Therefore, the energy consumption of the heat pump is found to be at-least $12 \%$ more than any other strategy despite considering the same operation time for the heat pump. In addition, the time-shiftable loads are scheduled in the evening for this strategy, which also contributes to higher energy costs for this strategy as electricity is generally expensive during evening hours. On top of that, in this strategy, the real-time market is not considered. As a result, from the cumulative results for summer and winter week in Tables 5 and 6 it can be seen that the total energy consumption and the energy cost for this strategy are expectedly the highest compared to the proposed method (and all other scheduling strategies). As the proposed method utilizes the flexibilities of building loads and DER units to participate in both the DA and RT markets, the total energy cost of 
Table 6: Cumulative results of scheduling and bidding strategies for winter week

\begin{tabular}{llcccc}
\hline & \multicolumn{4}{c}{ Scheduling and bidding strategy } \\
\cline { 2 - 6 } & Strategy 1 & Strategy 2 & Strategy 3 & Strategy 4 & Proposed method \\
\hline DA demand bids $(\mathrm{kWh})$ & 901.53 & 795.49 & 331.44 & 620.13 & 701.09 \\
DA supply bids $(\mathrm{kWh})$ & - & 45.01 & 125.69 & 186.35 & 192.72 \\
Total DA energy cost $(\$)$ & 225.81 & 165.70 & 59.98 & 76.09 & 80.63 \\
Expected RT bids $(\mathrm{kWh})$ & - & & 101.42 & 214.30 & 230.55 \\
Expected imbalance cost $(\$)$ & - & 6.72 & 67.72 & 67.79 & 65.61 \\
Net energy cost $(\$)$ & 225.81 & 158.98 & 127.70 & 143.88 & 146.24 \\
Storage degradation cost $(\$)$ & 3.78 & 19.12 & 55.78 & 17.82 & 10.98 \\
Thermal discomfort cost $(\$)$ & 0 & 8.85 & 16.08 & 1.03 & 3.36 \\
Interruption cost $(\$)$ & 0 & 0 & 3 & 0 & 0 \\
\hline
\end{tabular}

the proposed method is $51.09 \%$ lower in summer and $35.24 \%$ lower in winter than the inflexible strategy. However, the interruption cost and discomfort cost is zero for the inflexible strategy, as the flexibilities of time-shiftable and thermal loads are not considered. On the other hand, even though the interruption cost is zero for the proposed method as well, the flexible operation of the heat pump in the proposed method leads to some thermal discomfort for the user. Besides, the inflexible strategy does not contribute to any additional storage degradation cost other than standard calendar aging, whereas the proposed method's storage degradation cost is 2-3 times more than the inflexible strategy. However, the additional cost savings offered by the proposed method is much higher than the additional thermal discomfort and storage degradation cost.

2. Strategy 2 vs proposed method

The main difference between the second strategy and the proposed method is the additional cost-term added to the objective function of the proposed method to incorporate the uncertainties. The deterministic approach of strategy 2 schedules the building loads and DER units in day-ahead according to the forecast information of fixed-load demand, PV generation and DA market prices. According to this schedule, the day-ahead energy cost is found to be $\$ 93.61$ AUD for the summer week and $\$ 165.70$ AUD for the winter week. As the imbalances from the day-ahead schedules are considered to be traded in the RT market, the deviation from the forecasts would lead to an RT trading cost of $\$ 1.26$ AUD and \$6.72 AUD for the summer and winter week respectively. On the contrary, the net energy cost according to the proposed method is $21.39 \%$ lower for the summer and week and $8.01 \%$ lower for the winter week. The main reason behind this is that the stochastic approach of the proposed method considers the uncertainties and the expected cost of RT market trading while determining the schedule in the day-ahead stages. Therefore, it submits conservative DA demand and supply quantity bids compared to the deterministic method when uncertainty is higher, as shown in Fig. 11 . which shows the demand-supply quantity bids for a day according to the deterministic and stochastic methods. On the other hand, the deterministic method completely disregards the expected cost of RT trading and only considers the DA trading, whereas the stochastic method utilizes the lower tariffs of both DA and RT markets to schedule energy-extensive loads and reduces energy cost. However, the forecast prices of the RT market can also be considered for the day-ahead deterministic scheduling, which would lead to additional cost reductions thereby making this approach comparable to the proposed method. Besides, the deterministic schedule allows the prosumer to avoid higher price fluctuations in the RT market. However, the proposed stochastic method would outperform the deterministic method when uncertainties are higher as seen from the results.

3. Strategy 3 vs proposed method

The third strategy also incorporates the uncertainties while determining the day-ahead schedules considering both the DA and RT energy trading. However, the main difference between this strategy and the proposed method is that the proposed method also minimizes the storage degradation, thermal discomfort, and load interruptions. From the cumulating results in Tables 5 and 6 it can be seen that the net energy cost is lower in strategy 3 compared to the proposed method (and all other strategies as well). However, these significant cost reductions come with additional thermal discomfort, load interruptions, and storage degradation, which are highest for this strategy compared to all other strategies. The penalty cost for the interruption and thermal discomfort are virtual costs, not necessarily affect the actual financial savings of the prosumers. This is also true for the frequent charge-discharge penalty cost for the storage units. However, the cycle-aging degradation cost of the storage units is the actual financial cost that leads to depreciation of the storage units. From the simulation result, it is found that the third strategy provides a total of $\$ 38.87$ AUD additional energy cost savings compared to the proposed method for the two weeks (one summer and one winter week). However, it comes with an additional cycle-aging storage degradation of \$62.73 AUD compared to the proposed method. Therefore, such a scheduling strategy is not feasible considering the overall cost.

4. Strategy 4 vs proposed method

Contrary to the proposed method, the day-ahead scheduling model is formulated as a multi-objective optimization problem in strategy 4 and solved using the weighted-sum method, where equal weights are assigned for each objective 


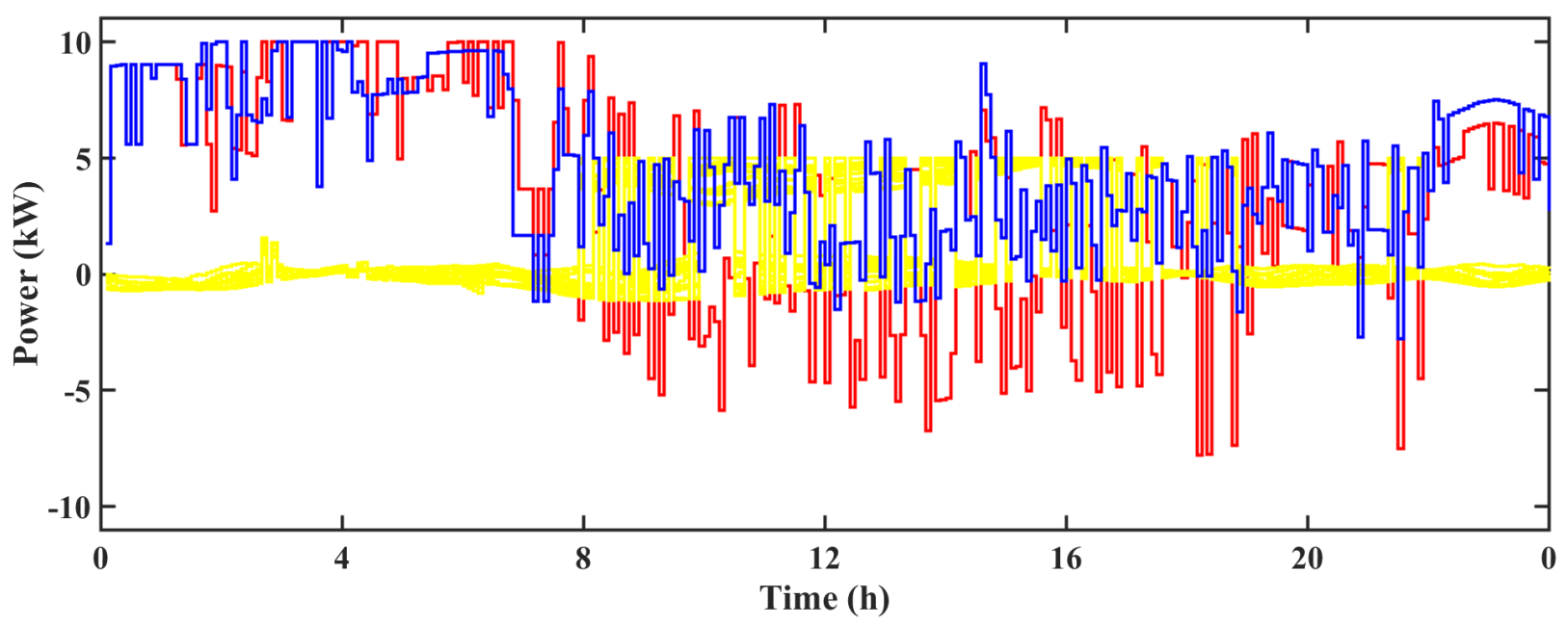

—DA bids (proposed method) — RT bid scenarios (proposed method) -DA energy bids (deterministic)

Figure 11: Demand and supply quantity bids according to the deterministic method vs stochastic method

after normalizing them with respect to their maximum and minimum values. From the cumulative results in Tables 5 and 6 it can be seen that the net energy cost of this strategy is almost similar to the proposed method for the summer and winter week. The load interruption costs are 0 for both strategy 4 and the proposed method. However, the multi-objective formulation of strategy 4 provides better results in terms of thermal discomfort, whereas the proposed method outperforms this multi-objective strategy in terms of storage degradation. As the storage degradation and net energy cost are the main factors indicating actual financial savings of the scheduling strategy, it can be argued that the proposed method provides better overall savings compared to the multi-objective method with equal weights. However, the weighted-sum results shown in Tables 5 and 6 represent only one optimal solution from the Pareto font. Any other point in the Pareto font would indicate different results, which can provide worse savings with regards to one objective(s) compared to the proposed method while outperforming the proposed method in terms of another objective (s). Therefore, such multi-objective based formulation is highly dependent on the weighing combinations used to get an optimal solution from the Pareto font. On the other hand, the proposed bi-level method offers overall better results (or similar for some objective(s)) in terms of actual financial cost savings for the prosumer as it provides only one global optimal solution that leads to minimum energy cost subject to minimum storage degradation, thermal discomfort, and load interruptions.

\subsection{Discussion}

A BEMS is presented in this paper with a bi-level optimization-based day-ahead scheduling model. The proposed BEMS utilizes the flexibilities of controllable building loads and DERs to schedule them in day-ahead to minimize the electricity cost for the prosumer. The proposed method is validated by simulation studies for a prosumer in Sydney, Australia. The simulation is run for a warmer week in summer and a colder week in winter. From the simulation results of device schedules in Section 4.4.2 it can be noticed that the prosumers' inconveniences and discomforts are also minimized while determining their schedule. For example, the flexible loads are scheduled in such a way that the user preferences in terms of desired operation windows are satisfied. Besides, none of the flexible loads are interrupted to minimize the interruption cost. This paper proposes a thermal model with VESS for the operation of the heat pump for space heating and cooling. From the schedule of the heat pump, it can be seen that the heat pump schedules maintain the indoor temperature within a flexible range. The VESS utilizes the thermal mass of the building to store energy virtually by prolonging the operation of the heat pump during the periods when electricity is cheaper or when DERs are available to supply the heat pump demand. This stored energy is later used by discharging the VESS to provide heating or cooling demand when electricity is expensive. The accuracy of the proposed thermal model in terms of heat pump demand estimation is verified by comparing the results of the proposed methodology with the thermal load demand calculated using the EnergyPlus. It is found that the heat pump demand does not deviate more than $4 \%$ of the values obtained using EnergyPlus. This flexible operation of a heat pump allows the BEMS to provide a significant reduction in heat pump power demand (simulation indicates a $10-22 \%$ reduction). From the cumulative results in Tables 5 and 6 it can be seen that the this cost reduction introduces thermal discomfort for the user. With a discomfort coefficient of $5 \mathrm{c} /{ }^{\circ} \mathrm{C}$, the total thermal discomfort cost is found to be $\$ 8.62$ AUD and $\$ 3.36$ AUD for the summer and winter week respectively. However, simulation results indicate that even with such discomfort the indoor temperature stays close to the desired set-point and fluctuates within user-specified limits. Hence, it can be argued that inconveniences and discomfort are 
minimized subject to user-specified flexibility for the reduction of energy cost. Simulation results also indicate that the schedule of storage units maintains the operational constraints and user preferences. For example, the EV battery is charged more than the user-specified SoC level before leaving home in the morning. Besides, the subsequent and frequent charge-discharge cycles are not completely prevented as this would reduce the cost savings for the prosumer, rather it is optimally reduced so that maximum cost savings can be achieved subject to minimum battery degradations.

The effectiveness of the proposed method is also compared with a few state-of-the-art day-ahead scheduling strategies. Case studies indicate that the proposed method can provide up to $51 \%$ cost savings for the prosumer compared to a worst-case non-optimal inflexible strategy, which does not optimize energy usage for cost minimization. The proposed method formulates the day-ahead scheduling model as a stochastic bi-level minimization problem that determines demand and supply quantity bids for the day-ahead and real-time markets by minimizing the day-ahead energy cost while factoring the expected cost of realtime imbalance cost for different uncertain scenarios. The case studies indicate that the proposed stochastic method is capable of weighing in expected cost for real-time energy trading for uncertain scenarios of fixed-load demand, PV generation, and real-time market prices. Therefore, it submits more conservative day-ahead energy-quantity bids compared to a deterministic scheduling strategy when the uncertainty is higher and utilizes the price elasticity in both markets to reduce energy costs. As a result, the proposed method provides up to $22 \%$ cost savings compared to deterministic scheduling strategy. The cost minimization problem is formulated as a bi-level optimization problem in the proposed method that minimizes the energy cost in the upper-level and a number of lower-level subproblems minimize storage degradation, load interruption, and thermal discomfort for the user. Case studies indicate that when the scheduling strategy only considers the energy cost it provides up to $28 \%$ additional energy cost savings for the prosumer, however, this also degrades the storage units and causes thermal discomfort for the user. From the cumulative results of the summer and winter week, it is found that the additional storage degradation caused by such scheduling strategy is more than the additional cost savings this method offers. In addition to that, the cost savings of the proposed method is also compared with a multi-objective optimization-based scheduling strategy. The multi-objective method is solved with equal weights for normalized objectives with respect to their individual maximum and minimum value for the same set of constraints. Even though it only provides one optimal solution (out of many in Pareto font) of the multi-objective method, the obtained cumulative energy cost, storage degradation, thermal discomfort, and load interruptions are compared with the proposed bi-level method. Case studies indicate that the proposed method performs better for some objectives while providing similar or slightly lesser results for other objectives. However, unlike the multi-objective method, the proposed bi-level method does not need to be tuned to get optimal results for each objective and can provide an optimal solution for minimum energy cost subject to minimum storage degradation, load interruption and thermal discomfort for the user.

However, the proposed method considers the prosumers as price-taker and only the energy-quantity bids are determined by the proposed scheduling methodology. To represent the actual transactive market model, the cost-bidding is also needed to be incorporated into the bidding model along with the quantity bids. Besides, the market competitiveness of the local real-time market is not considered in this paper. Another limitation of the proposed methodology is the simplification and reformulation of the non-linear bi-level problem in Eq. 24 as a MILP minimization problem in favor of reduced computation time. However, results indicate that this solving approach provides a near accurate solution with an RMSE of less than $0.009 \%$ for the piece-wise linearization. In addition to that, this paper only considers three uncertain sources and their scenarios are reduced to 20 for reducing the computation time. Future researches should include other sources of uncertainties with larger scenario set, such as EV availability, EV SoC at arrival, wholesale market prices, temperature, etc. Nonetheless, simulation and case studies indicate promising results of the proposed method in terms of actual financial cost savings for the prosumer when it participates in two-stage energy markets.

\section{Conclusion}

This paper proposes a BEMS model for residential prosumer that schedules the building loads and DER units in the day-ahead stage by minimizing day-ahead energy commitment costs while considering the expected cost of real-time imbalance trading for uncertain scenarios of fixed-load demand, PV generation, and market prices. The day-ahead scheduling model is formulated as a stochastic bi-level minimization problem that determines the energy quantity bids for the day-ahead and real-time markets by minimizing the energy cost in the upper-level, while a number of lower-level subproblems minimize inconveniences for the prosumer. Three types of controllable building devices are considered- flexible loads with time-shifting capabilities, storage units and thermal loads. All controllable building devices are scheduled according to user preferences and operational constraints. Comprehensive models are presented for all controllable building loads and DER units, where the user's inconveniences are represented as interruption costs for time-shiftable flexible loads, degradation costs for storage units, and thermal discomfort costs for the thermal loads. The proposed methodology is evaluated via simulation and case studies for a residential prosumer in Syndey, Australia for a summer and winter week. Case studies and simulation results indicate that the proposed BEMS can effectively satisfy all the user preferences and constraints for scheduling building loads and DERs. Results also indicate that the proposed method provides up to $51 \%$ more energy cost savings compared to non-optimal inflexible scheduling strategies, and can provide as high as $22 \%$ more cost savings compared to deterministic scheduling method. On the other hand, the proposed bi-level method offers better overall financial savings compared to the equally-weighted-sum multi-objective method.

However, the proposed BEMS models the prosumer as price-taker and does not consider the competitiveness of the local real-time market. Therefore, a comprehensive market simulation is essential with an appropriate market clearing mechanism and cost-bidding strategy to determine the market competitiveness and dispatch, which will be considered by the authors in 
future researches. Further researches will also be directed towards incorporating real-time local trading for power grid support services.

\section{References}

[1] B. Zhou, W. Li, K. W. Chan, Y. Cao, Y. Kuang, X. Liu, X. Wang, Smart home energy management systems: Concept, configurations, and scheduling strategies, Renewable and Sustainable Energy Reviews 61 (2016) 30-40. doi:10.1016/j. rser.2016.03.047.

[2] X. Jin, K. Baker, D. Christensen, S. Isley, Foresee: A user-centric home energy management system for energy efficiency and demand response, Applied Energy 205 (June) (2017) 1583-1595. doi:10.1016/j.apenergy.2017.08.166

[3] K. X. Perez, M. Baldea, T. F. Edgar, Integrated HVAC management and optimal scheduling of smart appliances for community peak load reduction, Energy and Buildings 123 (2016) 34-40. doi:10.1016/j.enbuild.2016.04.003

[4] GridWise Architecture Council, GridWise Transactive Energy Framework version 1, Tech. rep. (2015). doi:PNNL-22946 URL https://goo.gl/5oM4HG

[5] Pacific Northwest Smart Grid Demonstration Project: Technology Performance Report Highlights, Tech. rep. (2015). URL https://goo.gl/jrkvVk

[6] P. N. N. Laboratory, Pacific Northwest GridWise(TM) Testbed Demonstration Projects Part I . Olympic Peninsula Project Tech. rep. (2007). doi:10.2172/926122 URL https://goo.gl/Ym5nZb

[7] P. H. Divshali, B. J. Choi, H. Liang, Multi-agent transactive energy management system considering high levels of renewable energy source and electric vehicles, IET Generation, Transmission \& Distribution 11 (15) (2017) 3713-3721. doi:10.1049/iet-gtd.2016.1916.

[8] A. Lüth, J. M. Zepter, P. Crespo del Granado, R. Egging, Local electricity market designs for peer-to-peer trading: The role of battery flexibility, Applied Energy 229 (May) (2018) 1233-1243. doi:10.1016/j.apenergy.2018.08.004

[9] M. N. Akter, M. A. Mahmud, A. M T Oo, A Hierarchical Transactive Energy Management System for Microgrids, Energies 10 (12). doi:10.3390/en10122098

[10] O. Abrishambaf, F. Lezama, P. Faria, Z. Vale, Towards transactive energy systems: An analysis on current trends, Energy Strategy Reviews 26. doi:https://doi.org/10.1016/j.esr.2019.100418 URL http://www.sciencedirect.com/science/article/pii/S2211467X19301105

[11] M. Nizami, A. Haque, P. Nguyen, M. Hossain, On the application of home energy management systems for power grid support, Energy 188 (2019) 116104. doi:https://doi.org/10.1016/j.energy.2019.116104

[12] N. G. Paterakis, O. Erdinç, A. G. Bakirtzis, J. P. Catalão, Optimal household appliances scheduling under day-ahead pricing and load-shaping demand response strategies, IEEE Transactions on Industrial Informatics 11 (6) (2015) 15091519. doi:10.1109/TII.2015.2438534

[13] Y. Huang, H. Tian, L. Wang, Demand response for home energy management system, International Journal of Electrical Power and Energy Systems 73 (2015) 448-455. doi:10.1016/j.ijepes.2015.05.032.

[14] F. Luo, W. Kong, G. Ranzi, Z. Y. Dong, Optimal home energy management system with demand charge tariff and appliance operational dependencies, IEEE Transactions on Smart Grid (2019) 1-1doi:10.1109/TSG.2019.2915679

[15] R.-S. Liu, Y.-F. Hsu, A scalable and robust approach to demand side management for smart grids with uncertain renewable power generation and bi-directional energy trading, International Journal of Electrical Power and Energy Systems 97 (2018) 396 - 407. doi:https://doi.org/10.1016/j.ijepes.2017.11.023

URL http://www.sciencedirect.com/science/article/pii/S0142061517315995

[16] S. Nan, M. Zhou, G. Li, Optimal residential community demand response scheduling in smart grid Applied Energy 210 (2018) 1280 - 1289. doi:https://doi.org/10.1016/j.apenergy.2017.06.066 URL http://www.sciencedirect.com/science/article/pii/S030626191730819X

[17] S. Althaher, P. Mancarella, J. Mutale, Automated demand response from home energy management system under dynamic pricing and power and comfort constraints, IEEE Transactions on Smart Grid 6 (4) (2015) 1874-1883. doi:10.1109/TSG. 2014.2388357

[18] S. Zhou, F. Zou, Z. Wu, W. Gu, Q. Hong, C. Booth, A smart community energy management scheme considering user dominated demand side response and p2p trading International Journal of Electrical Power and Energy Systems 114 (2020) 105378. doi:https://doi.org/10.1016/j.ijepes.2019.105378 URL http://www.sciencedirect.com/science/article/pii/S0142061518339164

[19] M. S. H. Nizami, J. Hossain, E. Fernandez, Multi-agent based transactive energy management systems for residential buildings with distributed energy resources, IEEE Transactions on Industrial Informatics (2019) 1-12doi:10.1109/TII. 2019.2932109

[20] H. Hao, C. D. Corbin, K. Kalsi, R. G. Pratt, Transactive Control of Commercial Buildings for Demand Response, IEEE Trans. Power Syst. 32 (1) (2017) 774-783. doi:10.1109/TPWRS.2016.2559485

[21] S. Behboodi, D. P. Chassin, N. Djilali, C. Crawford, Transactive control of fast-acting demand response based on thermostatic loads in real-time retail electricity markets, Applied Energy 210 (2018) 1310 - 1320. doi:https://doi.org/10. 1016/j.apenergy.2017.07.058

URL http://www.sciencedirect.com/science/article/pii/S0306261917309315

[22] C. Long, J. Wu, Y. Zhou, N. Jenkins, Peer-to-peer energy sharing through a two-stage aggregated battery control in a community microgrid Applied Energy 226 (2018) 261 - 276. doi:https://doi.org/10.1016/j.apenergy.2018.05.097 URL http://www.sciencedirect.com/science/article/pii/S0306261918308146 
[23] M. S. H. Nizami, M. J. Hossain, K. Mahmud, J. Ravishankar, Energy cost optimization and der scheduling for unified energy management system of residential neighborhood, in: 2018 IEEE International Conference on Environment and Electrical Engineering and 2018 IEEE Industrial and Commercial Power Systems Europe (EEEIC / I CPS Europe), 2018, pp. 1-6. doi:10.1109/EEEIC.2018.8493732

[24] B. Celik, R. Roche, D. Bouquain, A. Miraoui, Decentralized neighborhood energy management with coordinated smart home energy sharing, IEEE Trans. Smart Grid PP (99). doi:10.1109/TSG.2017.2710358

[25] G. Prinsloo, A. Mammoli, R. Dobson, Customer domain supply and load coordination: A case for smart villages and transactive control in rural off-grid microgrids, Energy 135 (2017) 430-441. doi:10.1016/j.energy.2017.06.106

[26] C. Zhang, J. Wu, Y. Zhou, M. Cheng, C. Long, Peer-to-peer energy trading in a microgrid, Applied Energy 220 (2018) 1 - 12. doi:https://doi.org/10.1016/j.apenergy.2018.03.010. URL http://www.sciencedirect.com/science/article/pii/S0306261918303398

[27] B. Xu, J. Zhao, T. Zheng, E. Litvinov, D. S. Kirschen, Factoring the Cycle Aging Cost of Batteries Participating in Electricity Markets, IEEE Trans. Power Syst. 8950 (2017) 1-12. arXiv:1707.04567 doi:10.1109/TPWRS.2017.2733339

[28] Y. Cui, Z. Geng, Q. Zhu, Y. Han, Review: Multi-objective optimization methods and application in energy saving, Energy 125 (2017) 681 - 704. doi:https://doi.org/10.1016/j.energy.2017.02.174 URL http://www.sciencedirect.com/science/article/pii/S0360544217303584

[29] J. Iria, F. Soares, M. Matos, Optimal supply and demand bidding strategy for an aggregator of small prosumers, Applied Energy 213 (2018) 658 - 669. doi:https://doi.org/10.1016/j.apenergy.2017.09.002 URL http://www.sciencedirect.com/science/article/pii/S0306261917312758

[30] J. Iria, F. Soares, A cluster-based optimization approach to support the participation of an aggregator of a larger number of prosumers in the day-ahead energy market Electric Power Systems Research 168 (2019) 324 - 335. doi:https: //doi.org/10.1016/j.epsr.2018.11.022 URL http://www.sciencedirect.com/science/article/pii/S0378779618303973

[31] J. Soares, B. Canizes, M. A. F. Ghazvini, Z. Vale, G. K. Venayagamoorthy, Two-stage stochastic model using benders' decomposition for large-scale energy resource management in smart grids, IEEE Transactions on Industry Applications 53 (6) (2017) 5905-5914. doi:10.1109/TIA.2017.2723339

[32] S. Chen, C.-C. Liu, From demand response to transactive energy: state of the art, Journal of Modern Power Systems and Clean Energy 5 (1) (2017) 10-19. doi:10.1007/s40565-016-0256-x

[33] J. L. Dréau, P. Heiselberg, Energy flexibility of residential buildings using short term heat storage in the thermal mass Energy 111 (2016) 991 - 1002. doi:https://doi.org/10.1016/j.energy.2016.05.076 URL http://www.sciencedirect.com/science/article/pii/S0360544216306934

[34] S. Dempe, A. Zemkoho, On the karush-kuhn-tucker reformulation of the bilevel optimization problem, Nonlinear Analysis: Theory, Methods and Applications 75 (3) (2012) 1202 - 1218, variational Analysis and Its Applications. doi:https: //doi.org/10.1016/j.na.2011.05.097 URL http://www.sciencedirect.com/science/article/pii/S0362546X1100407X

[35] Tesla Powerwall 2 specifications URL https://www.tesla.com/en_AU/powerwall

[36] E. Fernandez, M. Hossain, M. Nizami, Game-theoretic approach to demand-side energy management for a smart neighbourhood in Sydney incorporating renewable resources, Applied Energy 232 (December) (2018) 245-257. doi: 10.1016/j.apenergy.2018.09.171

[37] GAMS/Cplex URL https://www.gams.com/latest/docs/S_CPLEX.html

[38] Smart-Grid Smart-City Electric Vehicle Trial Data URL https://data.gov.au/dataset/ds-dga-87f276c3-5fba-4f31-9032-199793d6f4a7/details

[39] EnergyPlus ${ }^{\text {IM }}$ URL https://energyplus.net/

[40] T. Yang, X. Zhang, Benchmarking the building energy consumption and solar energy trade-offs of residential neighborhoods on chongming eco-island, china, Applied Energy 180 (2016) 792-799. doi:https://doi.org/10.1016/j.apenergy.2016. 08.039

URL http://www.sciencedirect.com/science/article/pii/S0306261916311266

[41] V. Shabunko, C. Lim, S. Mathew, Energyplus models for the benchmarking of residential buildings in brunei darussalam. Energy and Buildings 169 (2018) 507 -516. doi:https://doi.org/10.1016/j.enbuild.2016.03.039.

URL http://www.sciencedirect.com/science/article/pii/S0378778816301931

[42] A. Konak, D. W. Coit, A. E. Smith, Multi-objective optimization using genetic algorithms: A tutorial Reliability Engineering and System Safety 91 (9) (2006) 992 - 1007, special Issue - Genetic Algorithms and Reliability. doi:https://doi.org/10.1016/j.ress.2005.11.018

URL http://www.sciencedirect.com/science/article/pii/S0951832005002012 